\title{
Radiative falloff of a scalar field in a weakly curved spacetime without symmetries
}

\author{
Eric Poisson \\ Department of Physics, University of Guelph, Guelph, Ontario, Canada N1G 2W1 and \\ Perimeter Institute for Theoretical Physics, 35 King Street North, Waterloo, Ontario, Canada N2J 2W9*
}

(Dated: April 29, 2002)

\begin{abstract}
We consider a massless scalar field propagating in a weakly curved spacetime whose metric is a solution to the linearized Einstein field equations. The spacetime is assumed to be stationary and asymptotically flat, but no other symmetries are imposed - the spacetime can rotate and deviate strongly from spherical symmetry. We prove that the late-time behavior of the scalar field is identical to what it would be in a spherically-symmetric spacetime: it decays in time according to an inverse power-law, with a power determined by the angular profile of the initial wave packet (Price falloff theorem). The field's late-time dynamics is insensitive to the nonspherical aspects of the metric, and it is governed entirely by the spacetime's total gravitational mass; other multipole moments, and in particular the spacetime's total angular momentum, do not enter in the description of the field's late-time behavior. This extended formulation of Price's falloff theorem appears to be at odds with previous studies of radiative decay in the spacetime of a Kerr black hole. We show, however, that the contradiction is only apparent, and that it is largely an artifact of the Boyer-Lindquist coordinates adopted in these studies.
\end{abstract}

\section{INTRODUCTION AND SUMMARY}

This paper is concerned with the late-time behavior of radiative fields in stationary curved spacetimes, a topic that has been the subject of vigorous investigation in the last three decades. Here we take the spacetime to be that of an isolated body of mass $M$, and we take the metric to be stationary and asymptotically flat. We imagine that the radiative field (a classical, massless field of integer spin that propagates on the fixed background spacetime) is given suitable initial data on a hypersurface $t=0$, and we examine its evolution at times $t$ much larger than $M$. Under these conditions, it is found that the field's late-time behavior is characterized by an inverse powerlaw decay. This property was first discovered by Richard Price [1], and it has been extensively studied since. This paper makes an additional contribution to this topic's vast literature by generalizing the formulation of Price's falloff theorem: we show that it holds under less restrictive conditions than are normally assumed. Although our considerations here will be restricted to a scalar field, much of what we shall say should apply equally well to an electromagnetic field, or to a (linearized) gravitationalperturbation field.

\section{Radiative falloff in spherical spacetimes}

Excluding the more recent work on radiative decay in the Kerr spacetime, which we review below, all previous studies of the late-time dynamics of massless fields were restricted to spherically-symmetric spacetimes. Here the field admits a decomposition in terms of spherical harmonics, and the field equations reduce to a one-

\footnotetext{
*Affiliate member
}

dimensional wave equation with an effective potential. For example, the evolution of a massless scalar field $\Psi$ in Schwarzschild spacetime is governed by the reduced wave equation

$$
\left[-\frac{\partial^{2}}{\partial t^{2}}+\frac{\partial^{2}}{\partial r^{* 2}}-V(r)\right] \psi_{l}(t, r)=0
$$

where $r^{*}=r+2 M \ln (r / 2 M+1)$ is the usual tortoise coordinate, $V(r)=(1-2 M / r)\left[l(l+1) / r^{2}+\right.$ $\left.2 M / r^{3}\right]$ is the effective potential, and $\psi_{l}(t, r)$ is a dimensionally-reduced field from which the scalar field can be reconstructed. The relationship is $r \Psi(t, r, \theta, \phi)=$ $\sum_{l m} a_{l m} \psi_{l}(t, r) Y_{l}^{m}(\theta, \phi)$, where $a_{l m}$ are amplitudes determined by the initial data, and $Y_{l}^{m}(\theta, \phi)$ are the standard spherical-harmonic functions. With a small change to the effective potential, $2 M / r^{3}$ going to $2\left(1-s^{2}\right) M / r^{3}$, the wave equation of Eq. (1.1) can be related to a massless field of (integer) spin $s[1,2]$, and this generalized potential can be shown to lead to a universal late-time behavior for $\psi_{l}$; conclusions based on the scalar-field equation therefore apply to fields of higher spin.

In his original treatment [1], Price dealt with the partial differential equation of Eq. (1.1) and solved it as a perturbative expansion in powers of $M$. This method was extended in Refs. [3, 4]. These works reveal that for times much larger than $r$ (which can be taken to be much larger than $M), \psi_{l}$ decays in time according to $t^{-(2 l+3)}$, assuming that the field's initial data has compact support and is not time-symmetric. This limit corresponds to approaching $t=\infty$ keeping $r$ finite; this gives the field's behavior near future timelike infinity. Another interesting limit corresponds to setting $v \equiv t+r^{*}=\infty$ keeping $u \equiv t-r^{*}$ finite; this gives the field's behavior at future null infinity. In this limit $\psi_{l}$ behaves as $u^{-(l+2)}$. The behavior of the field on the event horizon was also determined $[3,4]$, but we shall not discuss this here.

In an alternative strategy, Leaver [5] performed a Fourier transform (in $t$ ) of Eq. (1.1) and examined the re- 
sulting ordinary differential equation (in $\left.r^{*}\right)$. He showed that the inverse power-law decay of the field is associated with a branch-cut discontinuity of the solutions in the complex frequency plane, and he reproduced the correct late-time behavior with a low-frequency approximation ( $M \omega \ll 1$, where $\omega$ denotes frequency) to the differential equation. The low-frequency regime is naturally related to the field's late-time dynamics $(t \gg M)$, and to the asymptotic behavior of the effective potential $(r \gg M)$. This method was generalized in Refs. [6-8].

The work of Ching et al. [6] is especially noteworthy, because it contributes a very simple heuristic understanding of the inverse power-law decay. These authors show that the late-time field at the spatial position $r$ is produced by the part of the initial wave packet that propagates outward to a distant point $r^{\prime \prime}$ (such that $r^{\prime \prime}$ is much larger than $r$ ), scatters off a small amount of spacetime curvature there, and makes its way back to $r$ after a time $t \simeq 2 r^{\prime \prime}$. They show further that if $\delta V(r) \equiv V(r)-l(l+1) / r^{* 2}$ is the part of the effective potential that can be directly associated with spacetime curvature, then the late-time field is proportional to $\delta V(t / 2)$; this holds for arbitrary potentials $V(r)$. The inverse power-law nature of the effective potential naturally gives rise to an inverse power-law decay for the field.

The work reviewed above reveals that the late-time behavior of a radiative field is governed only by the asymptotic form of the metric at large distances from the gravitating body. In this sense, the radiative falloff brings information about the spacetime's asymptotic structure to an observer located much closer to the gravitating body. On the other hand, the late-time dynamics of a massless field reveals nothing of the nature of the central object, which might be extremely diffuse or extremely compact. While the body's internal structure certainly affects the field at early times [7, 9-17], the radiative falloff depends only on the asymptotic conditions. The robustness of the inverse power-law decay is well demonstrated in Refs. [18-20], in which numerical simulations of the collapse of a self-gravitating scalar field were carried out; the decay is seen at late times whether or not the collapse produces a black hole. That the late-time dynamics of a massless field is directly coupled to the spacetime's asymptotic structure is well illustrated in Refs. [21-27], in which different asymptotic conditions are imposed, and corresponding changes in late-time behavior observed. Changes also occur if the simple massless fields considered here are replaced by more exotic fields; this was explored in Refs. [28-32].

\section{New formulation of Price's falloff theorem}

Our previous conclusion, that the late-time dynamics of a massless field is governed only by the spacetime's asymptotic structure, and is independent of the details of the metric near the center of mass, is based on calcu- lations that assume spherical symmetry. But this observation suggests that the assumption is not a severe restriction, as any stationary, asymptotically-flat spacetime becomes approximately spherical at large distances from the gravitating body. We would therefore expect that the picture of radiative falloff painted previously should not change qualitatively if the assumption of spherical symmetry were to be removed. Our main purpose with this paper is to prove that this expectation is warranted: We investigate the radiative falloff of a massless scalar field in a nonspherical spacetime, and show that the same picture emerges.

The observation also suggests that in order to study the late-time dynamics of a massless field, it should be sufficient to examine its propagation in a spacetime that is only weakly curved, since this condition is necessarily met in the asymptotic region. This, then, defines our approach: We look at the late-time evolution of a massless scalar field $\Psi$ in a stationary, asymptotically-flat spacetime whose metric satisfies the Einstein field equations linearized about flat spacetime.

The metric is expressed as

$d s^{2}=-(1+2 \Phi) d t^{2}+(1-2 \Phi)\left(d x^{2}+d y^{2}+d z^{2}\right)+8(\boldsymbol{A} \cdot d \boldsymbol{x}) d t$,

in terms of a scalar potential $\Phi(\boldsymbol{x})$ and a vector potential $\boldsymbol{A}(\boldsymbol{x})$, in which $\boldsymbol{x}=(x, y, z)$ denotes a quasi-Cartesian frame adapted to the underlying flat spacetime. The potentials do not possess any (spatial) symmetries, but it is assumed that they do not depend on time $t$. The matter distribution can thus be arbitrarily distorted, but it is assumed to be stationary and bounded by a sphere of radius $\mathcal{R}$. The spacetime is therefore asymptotically flat, and the potentials outside the matter distribution are characterized by a (potentially infinite) number of mass and current multipole moments. Details regarding the metric and the potentials are presented in Sec. II. Details regarding the equation satisfied by the scalar field are given in Sec. III. Throughout the paper we work consistently to first order in the gravitational potentials, and we use units such that $G=c=1$.

From the quasi-Cartesian coordinates $(x, y, z)$ we form quasi-spherical coordinates $(r, \theta, \phi)$ by invoking the usual relations $x=r \sin \theta \cos \phi, y=r \sin \theta \sin \phi$, and $z=$ $r \cos \theta$. We then give the scalar field initial data

$$
\begin{aligned}
& \Psi\left(0, \boldsymbol{x}^{\prime}\right)=C\left(r^{\prime}\right) Y_{l}^{m}\left(\theta^{\prime}, \phi^{\prime}\right), \\
& \dot{\Psi}\left(0, \boldsymbol{x}^{\prime}\right)=\dot{C}\left(r^{\prime}\right) Y_{l}^{m}\left(\theta^{\prime}, \phi^{\prime}\right)
\end{aligned}
$$

on the hypersurface $t=0$. (The symbol $\boldsymbol{x}^{\prime}$ designates a point on the hypersurface.) Here, $C\left(r^{\prime}\right)$ represents the radial profile of the initial wave packet, and $\dot{C}\left(r^{\prime}\right)$ gives the profile of the field's velocity. [These functions are independent and the overdot should not suggest that $\dot{C}\left(r^{\prime}\right)$ is the time derivative of $C\left(r^{\prime}\right)$; it is just a convenient mnemonic that reminds us that while $C\left(r^{\prime}\right)$ is associated with the field's initial configuration, $\dot{C}\left(r^{\prime}\right)$ is associated with the field's initial velocity.] We assume that the 
field's initial data has compact support: the functions $C\left(r^{\prime}\right)$ and $\dot{C}\left(r^{\prime}\right)$ must vanish outside of a sphere of radius $\mathcal{L}$. This is an essential assumption: as we shall see, the methods developed in this paper cannot be directly applied to initial data that do not satisfy this property. As Eq. (1.3) indicates, we assume that the field's initial angular profile is described by a spherical-harmonic function of degree $l$ and azimuthal index $m$. This choice is largely arbitrary, because the absence of spherical symmetry in the background spacetime denies any special status to the spherical harmonics. They constitute, nevertheless, a convenient basis in which to decompose the field's initial angular profile. The additional choice of assigning the same spherical harmonic to both $\Psi\left(0, \boldsymbol{x}^{\prime}\right)$ and $\dot{\Psi}\left(0, \boldsymbol{x}^{\prime}\right)$ is also arbitrary, and it is made for the sake of simplicity.

The initial data of Eq. (1.3) is evolved forward in time in the weakly curved spacetime of Eq. (1.2). This is done by means of a retarded Green's function $G\left(t, \boldsymbol{x} ; t^{\prime}, \boldsymbol{x}^{\prime}\right)$ that we compute in Sec. IV. In that section we prove one of the central results of this paper: Despite the absence of spherical symmetry, for times $t>t^{\prime}+r+3 r^{\prime}+2 \mathcal{R}$ the Green's function is sensitive only to the sphericallysymmetric aspects of the metric; it is entirely oblivious to the vector potential $\boldsymbol{A}$ and the nonspherical part of the scalar potential $\Phi$. We show that for these times, the Green's function is given by

$$
G\left(t, \boldsymbol{x} ; t^{\prime}, \boldsymbol{x}^{\prime}\right)=-\frac{8 M\left(t-t^{\prime}\right)}{\left[\left(t-t^{\prime}\right)^{2}-\left|\boldsymbol{x}-\boldsymbol{x}^{\prime}\right|^{2}\right]^{2}},
$$

where $\left|\boldsymbol{x}-\boldsymbol{x}^{\prime}\right|$ is the Euclidean distance between $\boldsymbol{x}$ and $\boldsymbol{x}^{\prime}$, and $M$ is the total gravitational mass of the spacetime. Equation (1.4) indicates very clearly that only the monopole moment of the scalar potential enters in the description of the late-time Green's function; higher multipole moments play absolutely no role. It should be noted that in Sec. IV, the Green's function is calculated perturbatively through first order in the gravitational potentials; Eq. (1.4) is therefore valid up to terms quadratic in $\Phi$ and $\boldsymbol{A}$.

This remarkable property of the Green's function guarantees that in the weakly curved spacetime of Eq. (1.2), the late-time behavior of the scalar field will be the same whether or not the spacetime is spherically symmetric. While the early-time behavior brings out essential differences, wave propagation at late times is insensitive to any nonspherical aspect of the spacetime. The spherical picture of radiative falloff painted previously is therefore not just a good approximation, it is virtually exact, so long as we do not probe the nonlinear aspects of the problem (see below for a clarification of this qualifying statement).

By integrating the initial data of Eq. (1.3) against the Green's function of Eq. (1.4), we find in Sec. V that the scalar field's behavior near future timelike infinity $(t \gg r)$ is given by

$$
\Psi(t \gg r) \simeq 4 M(-1)^{l+1} \frac{(2 l+2) ! !}{(2 l+1) ! !}\left(\dot{C}_{l}-\frac{2 l+3}{t} C_{l}\right)
$$

$$
\times \frac{r^{l}}{t^{2 l+3}} Y_{l}^{m}(\theta, \phi)
$$

up to a fractional correction of order $r^{2} / t^{2}$. The quantities $C_{l}$ and $\dot{C}_{l}$ are moments of the field's initial radial profile; for example, $C_{l} \equiv \int C\left(r^{\prime}\right)\left(r^{\prime}\right)^{l+2} d r^{\prime}$. In Sec. V we also obtain the field's behavior at future null infinity. To get this we introduce the retarded time coordinate $u \equiv t-r$, the advanced time coordinate $v \equiv t+r$, and we take the limit $v \rightarrow \infty$. After multiplying the field by $r$ to produce a nonzero result, we find

$$
\begin{aligned}
r \Psi(v=\infty) \simeq & 2 M(-1)^{l+1} \frac{(l+1) !}{(2 l+1) ! !}\left(\dot{C}_{l}-\frac{l+2}{u} C_{l}\right) \\
& \times \frac{1}{u^{l+2}} Y_{l}^{m}(\theta, \phi)
\end{aligned}
$$

up to a fractional correction of order $\mathcal{L}^{2} / u^{2}$. These results agree precisely with those obtained previously for Schwarzschild spacetime $[3,5]$. In Sec. V we show that both relations can be obtained by taking a different limit of a more general description according to which $r \Psi \propto r^{l+1} t /\left(t^{2}-r^{2}\right)^{l+2} \propto(1-u / v)^{l+1}(1+u / v) / u^{l+2}$. The transition from a $t^{-(2 l+3)}$ behavior near future timelike infinity to a $u^{-(l+2)}$ behavior at future null infinity is thus naturally explained. To the best of our knowledge, the interpolating formula of Eq. (5.15), below, has not appeared before in the literature; that Eqs. (1.5) and (1.6) can be obtained by taking two different limits of a more general description appears to be a new result.

Our new statement of Price's falloff theorem is considerably more general than previous formulations, which were all restricted to spherically-symmetric spacetimes. We prove here that in a stationary, asymptotically-flat, and weakly-curved spacetime without spatial symmetries, the late-time behavior of a scalar field is identical to what it would be in a spherically-symmetric spacetime. We find that the late-time dynamics is insensitive to the nonspherical aspects of the metric, and that it is governed entirely by the spacetime's total gravitational mass; other multipole moments, and in particular the spacetime's total angular momentum, do not enter in the description of the field's late-time behavior. This conclusion is compatible with our previous intuition: Because the radiative falloff is governed by the asymptotic form of the gravitational field, and because the field must become increasingly spherical at large distances from the gravitating body, the spherical description should be reliable. What we show here, in effect, is that the spherical description is virtually exact, and that it is even more reliable than could have been expected.

\section{Radiative falloff in Kerr}

This conclusion appears to be at odds with previous studies of radiative decay in the spacetime of a Kerr black hole. This topic has been the subject of both analytical 
[33-38] and numerical [39-41] investigation. For the purpose of this discussion we rely mostly on the analytical description of the late-time behavior of a scalar field provided by Hod [37]. The most reliable numerical work also focused on the radiative falloff of a scalar field, and it was carried out by Krivan [41].

The late-time dynamics of a massless scalar field in the Kerr spacetime appears to be much more complicated than what is suggested by our preceding discussion. Supposing once more that the field's initial data is a pure spherical-harmonic mode $Y_{l}^{m}$, the field's behavior near future timelike infinity is found to be given by [37]

$$
\Psi \propto \begin{cases}Y_{l}^{m} / t^{2 l+3} & l=m \text { or } l=m+1 \\ Y_{m}^{m} / t^{l+m+1} & l-m \geq 2(\text { even }) \\ Y_{m+1}^{m} / t^{l+m+2} & l-m \geq 2(\text { odd })\end{cases}
$$

in apparent conflict with the result stated in Eq. (1.5). The Kerr results imply that an initial mode of degree $l$ does not necessarily survive at late times. In general it is converted into a mode of degree $m$ or $m+1$; this is the mode of smallest degree compatible with the initial value of $m$ (which does not change because of the axisymmetry of the Kerr spacetime) and with the parity of the initial mode (which is conserved during the evolution). Ignoring the fact that Eq. (1.7) does not agree with our previous predictions, that evolution in the Kerr spacetime should produce mode conversion is not necessarily surprising: the spacetime is not spherically symmetric, and spherical-harmonic modes should not be expected to evolve independently. It is therefore expected that an initial $Y_{l}^{m}$ mode should be converted to a $Y_{m}^{m}$ mode if $l-m$ is even, or to a $Y_{m+1}^{m}$ mode if $l-m$ is odd. What is surprising, however, is that a final $Y_{m}^{m}$ mode does not necessarily decay in time according to $t^{-(2 m+3)}$, which is what it should do according to our spherical-symmetry expectation; instead, the decay is given by $t^{-(l+m+1)}$ when $l \geq m+2$. Similarly, a final $Y_{m+1}^{m}$ mode does not decay according to $t^{-(2 m+5)}$, but goes instead as $t^{-(l+m+2)}$ when $l \geq m+2$.

The behavior displayed in Eq. (1.7) is loosely confirmed by Krivan's numerical work [41]. Krivan shows that an initial $Y_{4}^{0}$ mode is converted to a $Y_{0}^{0}$ mode during the evolution, and finds a decay described approximately by $t^{-5.5}$. This is much closer to Hod's prediction of $t^{-5}$ than to the naive (spherical-symmetry based) expectation of $t^{-3}$. Although the agreement is far from perfect, it clearly suggests that Eq. (1.7) is closer to the truth than Eq. (1.5), even after allowing for mode conversion.

The field's radiative falloff at future null infinity is even more complicated. Here Hod finds [37]

$r \Psi \propto \begin{cases}Y_{l}^{m} / u^{l+2} & l=m \text { or } l=m+1, \\ \left(Y_{m}^{m}, Y_{m+2}^{m}, \cdots Y_{l-2}^{m}\right) / u^{l} & l-m \geq 2 \text { (even), } \\ \left(Y_{m+1}^{m}, Y_{m+3}^{m}, \cdots Y_{l-2}^{m}\right) / u^{l} & l-m \geq 2 \text { (odd), }\end{cases}$

where the notation indicates that in general, the angular description of the late-time field involves a number of spherical-harmonic modes. The mode-conversion mechanism is clearly more complicated here, and the results of Eq. (1.8) disagree with Eq. (1.6); they also seem to be incompatible with our spherical-symmetry expectations.

Our new formulation of Price's falloff theorem makes an unambiguous statement: The late-time behavior of a scalar field in a stationary, asymptotically-flat, and weakly curved spacetime is governed only by the spherical aspects of the metric, and is not affected by any deviation from spherical symmetry. Provided that the strong-field aspects of the spacetime can truly be ignored, as we have argued, the theorem predicts that a scalar field in the Kerr spacetime should behave as predicted by Eq. (1.5) and (1.6). Instead we find the behavior of Eq. (1.7) and (1.8). Is there a contradiction between the two sets of results?

The answer is no - there is no contradiction. The key point is that the late-time dynamics of a scalar field in Kerr was examined in the Boyer-Lindquist coordinates, which give one of the simplest descriptions of the Kerr spacetime. But in these coordinates, the asymptotic form of the Kerr metric does not match the metric of Eq. (1.2), whose relevant spherical part is given by $d s^{2}=-(1-2 M / r) d t^{2}+(1+2 M / r)\left(d r^{2}+r^{2} d \theta^{2}+\right.$ $\left.r^{2} \sin ^{2} \theta d \phi^{2}\right)$. Indeed, even in the limit $M \rightarrow 0$ the Kerr metric does not coincide with the Minkowski metric expressed in spherical coordinates $(r, \theta, \phi)$. To produce a match it is necessary to introduce spheroidal coordinates $(\hat{r}, \hat{\theta}, \phi)$ defined by the relations $x=\sqrt{\hat{r}^{2}+e^{2}} \sin \hat{\theta} \cos \phi$, $y=\sqrt{\hat{r}^{2}+e^{2}} \sin \hat{\theta} \sin \phi$, and $z=\hat{r} \cos \hat{\theta}$, in which the constant $e$ measures the ellipticity of the coordinate system. Rewriting the Minkowski metric in terms of these coordinates produces agreement with the $M \rightarrow 0$ limit of the Kerr metric, provided that $e$ be identified with Kerr's rotation parameter: $e=J / M$, where $J$ is the spacetime's total angular momentum.

It is therefore the spheroidal coordinates, and not the original spherical coordinates, that constitute the appropriate weak-field limit of the Boyer-Lindquist coordinates. This implies that a meaningful comparison between the results of Eqs. (1.5), (1.6) and those of Eqs. (1.7), (1.8) must involve a transformation from the spherical coordinates $(r, \theta, \phi)$ - used to express the first set of results - to the spheroidal coordinates $(\hat{r}, \hat{\theta}, \phi)-$ implicitly used to express the second set of results. Stated differently, the initial data used in the context of Kerr has the form of $\hat{C}(\hat{r}) Y_{l}^{m}(\hat{\theta}, \phi)$, and this is substantially different from what is written in Eq. (1.3). Equations (1.7) and (1.8) refer to this different choice of initial conditions, and they do not agree with Eqs. (1.5) and (1.6) simply because the evolutions proceed from different initial data. The two sets of results therefore represent very different situations, and it is meaningless to compare them directly.

We elaborate this argument in the last two sections of the paper. In Sec. VI we retrace the computations of Sec. V, starting with initial conditions of the form of Eq. (1.3) but stated in terms of spheroidal coordinates. 
We evolve this initial data with the Green's function of Eq. (1.4), also expressed in terms of spheroidal coordinates. Our results for the late-time behavior of the scalar field are in perfect agreement with Hod's results [37] displayed in Eqs. (1.7) and (1.8). In Sec. VII we give a direct comparison between the descriptions of radiative falloff based on spheroidal and spherical coordinates, and show how they can be reconciled. The analysis presented in these two sections implies that the results of Eqs. (1.7) and (1.8) are largely an artifact of the Boyer-Lindquist coordinates, which obscure the effective spherical symmetry of the problem. It also shows that there is no contradiction between Hod's results and our generalized statement of Price's falloff theorem: the higher multipole moments of the Kerr spacetime, including its angular momentum, play no role in the description of the field's late-time dynamics.

\section{Open questions}

Because our calculations agree completely with Hod's [37], they shed no light on the apparent discrepancy between the analytical description of Eq. (1.7) and Krivan's numerical results [41]. (Recall that Krivan finds an initial $Y_{4}^{0}$ mode to decay approximately as $t^{-5.5}$, while Hod predicts $t^{-5}$ with an error of order $t^{-7}$.) A possible explanation for this discrepancy might be that Krivan's numerical results are sensitive to subdominant terms in the expansion of $\Psi$ in inverse powers of $t$; this would explain the slightly larger power. It is also possible that Krivan's results are revealing a nonlinear effect of the sort discussed by Hod in Ref. [42]. More work will be needed to clarify this issue.

It will also be important to better understand the limitations of our weak-field assumption. Consider the early-time evolution of a scalar field from $t=0$ to some time $t=t_{1}$. During this epoch the field is sensitive to the nonspherical aspects of the metric, and the initial data of Eq. (1.3) gets converted into a superposition of (potentially many) spherical-harmonic modes. Of these, the most relevant for the field's late-time behavior is the mode of lowest degree in $l$, because it is this mode that will dominate at late times. (For example, an initial $Y_{4}^{0}$ field should be expected to produce a $Y_{0}^{0}$ mode at $t=t_{1}$, and this should give rise to a field that decays as $t^{-3}$, not $t^{-11}$, at late times.) Isolating this mode, the field's new initial data at $t=t_{1}$ can once more be written as in Eq. (1.3), but with a potentially lower value of $l$, and with radial functions $C(r)$ and $\dot{C}(r)$ that are now explicitly proportional to higher multipole moments of the gravitational field. According to Eqs. (1.5) and (1.6), then, the field at late times will be dominated by a sphericalharmonic mode that might be different from the original - the final $l$ might be smaller than the initial $l$, in violation of our predictions.

This, however, is an effect that is clearly nonlinear in the gravitational potentials, as the amplitude of the field at late times is now found to be proportional to the product of $M$ times $C_{l}$ or $\dot{C}_{l}$, which are already proportional to higher multipole moments of these potentials. Our calculational methods do not allow us to describe such a nonlinear evolution of the scalar field, and we shall have to leave this issue unexplored for the time being. But while such a nonlinear effect could ultimately be important, we are not aware of any indication in the literature that it might already have been seen. Krivan's numerical results [41], for example, describe a radiative decay in Kerr that is slower, not faster, than what is expected on the basis of a linear analysis - he sees an initial $Y_{4}^{0}$ mode decaying as $t^{-5.5}$, not $t^{-3}$, while our linear prediction is $t^{-5}$.

With a modest effort, the work presented in this paper could be extended to the more relevant cases of electromagnetic and gravitational radiative fields. Methods to compute Green's functions for these fields were introduced in Refs. [43, 44] in the context of a weakly curved spacetime with vanishing vector potential $\boldsymbol{A}$, and these methods could easily be generalized to the situation at hand. While we can be reasonably sure that these fields will give rise to a very similar formulation of the generalized falloff theorem, a complete justification of this statement must await future work.

\section{Organization of the paper}

The technical part of the paper begins in Sec. II, where we present a detailed description of the stationary, asymptotically-flat, and weakly-curved spacetime in which the massless scalar field $\Psi$ is propagating; the metric for this spacetime was already displayed in Eq. (1.2). In Sec. III we write down the wave equation for the scalar field and present its solution in terms of a retarded Green's function. In Sec. IV we compute this Green's function and show that at late times, it reduces to the result of Eq. (1.4); some technical aspects of this computation are relegated to Appendix A. In Sec. V we calculate the late-time behavior of the scalar field and derive the results of Eqs. (1.5) and (1.6); technical details are relegated to Appendix B. In Sec. VI we switch from spherical coordinates $(r, \theta, \phi)$ to spheroidal coordinates $(\hat{r}, \hat{\theta}, \phi)$ and show that the transformation is responsible for the very different description of Eqs. (1.7) and (1.8); technical details are relegated to Appendix C. Finally, in Sec. VII we give a direct comparison between the two sets of initial data obtained in the two coordinate systems.

\section{Remarks on notation}

Throughout the paper we use geometrized units, in which $G=c=1$. In the text we often refer to three different points in spacetime, $x=(t, \boldsymbol{x}), x^{\prime}=\left(t^{\prime}, \boldsymbol{x}^{\prime}\right)$, and $x^{\prime \prime}=\left(t^{\prime \prime}, \boldsymbol{x}^{\prime \prime}\right)$. We let $\partial_{\alpha}=\left(\partial_{t}, \partial_{a}\right)$ denote partial differentiation with respect to $x^{\alpha}=\left(t, x^{a}\right), \partial_{\alpha^{\prime}}=\left(\partial_{t^{\prime}}, \partial_{a^{\prime}}\right)$ 
partial differentiation with respect to $x^{\prime \alpha}=\left(t^{\prime}, x^{\prime a}\right)$, and $\partial_{\alpha^{\prime \prime}}=\left(\partial_{t^{\prime \prime}}, \partial_{a^{\prime \prime}}\right)$ partial differentiation with respect to $x^{\prime \prime \alpha}=\left(t^{\prime \prime}, x^{\prime \prime a}\right)$. We let $\partial_{\alpha \beta} \equiv \partial_{\alpha} \partial_{\beta}, \partial_{\alpha^{\prime} \beta^{\prime}} \equiv \partial_{\alpha^{\prime}} \partial_{\beta^{\prime}}$, $\partial_{\alpha^{\prime \prime} \beta^{\prime \prime}} \equiv \partial_{\alpha^{\prime \prime}} \partial_{\beta^{\prime \prime}}$, or any other combination indicate repeated differentiation. To generalize this to any number of derivatives we introduce a multi-index $N \equiv a_{1} a_{2} \cdots a_{n}$ that contains a number $n$ of individual (spatial) indices - we shall need this only for differentiation with respect to the spatial coordinates. Thus, $\partial_{N} \equiv \partial_{a_{1} a_{2} \cdots a_{n}}$, $\partial_{N^{\prime}} \equiv \partial_{a_{1}^{\prime} a_{2}^{\prime} \cdots a_{n}^{\prime}}$, and $\partial_{N^{\prime \prime}} \equiv \partial_{a_{1}^{\prime \prime} a_{2}^{\prime \prime} \cdots a_{n}^{\prime \prime}}$.

\section{METRIC OF A WEAKLY CURVED SPACETIME}

We consider the weakly curved spacetime of a stationary matter distribution described by a mass density $\rho(\boldsymbol{x})$ and a mass-current density $\boldsymbol{j}(\boldsymbol{x}) \equiv \rho \boldsymbol{v}$, where $\boldsymbol{v}$ is the velocity field within the matter distribution. We use the bold symbol $\boldsymbol{x}$ to denote the spatial coordinates $(x, y, z)$, and a bold symbol like $\boldsymbol{v}$ to denote a vector in ordinary three-dimensional flat space; all vectorial operations shall refer to this space. [45]

The metric of such a spacetime can be expressed as

$d s^{2}=-(1+2 \Phi) d t^{2}+(1-2 \Phi)\left(d x^{2}+d y^{2}+d z^{2}\right)+8(\boldsymbol{A} \cdot d \boldsymbol{x}) d t$

where $\Phi$ and $\boldsymbol{A}$ are gravitational potentials defined by

$$
\Phi(\boldsymbol{x})=-\int \frac{\rho\left(\boldsymbol{x}^{\prime}\right)}{\left|\boldsymbol{x}-\boldsymbol{x}^{\prime}\right|} d^{3} x^{\prime}
$$

and

$$
\boldsymbol{A}(\boldsymbol{x})=-\int \frac{\boldsymbol{j}\left(\boldsymbol{x}^{\prime}\right)}{\left|\boldsymbol{x}-\boldsymbol{x}^{\prime}\right|} d^{3} x^{\prime}
$$

The metric of Eqs. (2.1)-(2.3) is a solution to the Einstein field equations linearized about flat spacetime; the source terms are defined in terms of the matter's stress-energy tensor by $\rho=T^{t t}$ and $j^{a}=T^{t a}$, where $a=1,2,3$ represents a spatial index. The metric is expressed in the (linearized) harmonic gauge, and the corresponding gauge condition for a stationary situation is $\boldsymbol{\nabla} \cdot \boldsymbol{A}=0$. This follows automatically from Eq. (2.3) and energy-momentum conservation, which produces the (time-independent) continuity equation $\boldsymbol{\nabla} \cdot \boldsymbol{j}=0$. Throughout the paper we will work consistently to first order in the potentials $\Phi$ and $\boldsymbol{A}$.

We suppose that the matter distribution is bounded by a sphere of radius $\mathcal{R}$. In the integrals of Eqs. (2.2) and (2.3), therefore, the source point $\boldsymbol{x}^{\prime}$ is such that $r^{\prime} \equiv\left|\boldsymbol{x}^{\prime}\right|$ never exceeds $\mathcal{R}$. If a gravitational potential is evaluated at a field point $\boldsymbol{x}$ such that $r \equiv|\boldsymbol{x}|$ is greater than $\mathcal{R}$, it can be expressed as a multipole expansion in powers of $1 / r$. Specifically,

$$
\Phi(\boldsymbol{x})=-\frac{M}{r}-\sum_{n=1}^{\infty} \frac{(-1)^{n}}{n !} M^{N} \partial_{N}\left(\frac{1}{r}\right)
$$

and

$$
A^{a}(\boldsymbol{x})=J^{a b} \partial_{b}\left(\frac{1}{r}\right)-\sum_{n=2}^{\infty} \frac{(-1)^{n}}{n !} J^{a N} \partial_{N}\left(\frac{1}{r}\right) .
$$

Here, $M=\int \rho\left(\boldsymbol{x}^{\prime}\right) d^{3} x^{\prime}$ is the total mass of the matter distribution, and $M^{N}=\int \rho\left(\boldsymbol{x}^{\prime}\right) x^{\prime N} d^{3} x^{\prime}$ are higher multipole moments of the mass density. The multiindex $N \equiv a_{1} a_{2} \cdots a_{n}$ includes a number $n$ of spatial indices, and we set $\partial_{N} \equiv \partial_{a_{1} a_{2} \cdots a_{n}} \equiv \partial_{a_{1}} \partial_{a_{2}} \cdots \partial_{a_{n}}$ and $x^{\prime N} \equiv x^{\prime a_{1}} x^{\prime a_{2}} \cdots x^{\prime a_{n}}$. We also have introduced $J^{a b}=\int j^{a}\left(\boldsymbol{x}^{\prime}\right) x^{\prime b} d^{3} x^{\prime}$ as the dipole moment of the current density, and $J^{a N}=\int j^{a}\left(\boldsymbol{x}^{\prime}\right) x^{\prime N} d^{3} x^{\prime}$ as its higher multipole moments. The dipole moment is intimately related to $\boldsymbol{J}$, the total angular momentum of the matter distribution; this is defined by $\boldsymbol{J}=\int \boldsymbol{x}^{\prime} \times \boldsymbol{j}\left(\boldsymbol{x}^{\prime}\right) d^{3} x^{\prime}$ and the relationship is $J^{a b}=-\frac{1}{2} \varepsilon^{a b c} J_{c}$, with $\varepsilon^{a b c}$ denoting the completely antisymmetric permutation symbol.

The multipole moments $M^{N}$ and $J^{a N}$ are all symmetric under permutations of individual indices within the multi-index $N$. Because any "pure-trace" quantity of the form $\delta^{a b} \partial \ldots a \cdots b \ldots(1 / r)$ vanishes away from $r=0$, only the tracefree part of each multipole moment is actually required in the expansions of Eqs. (2.4) and (2.5) [46]. For our purposes, however, it will not be necessary to explicitly remove the "trace part" of the multipole moments; as this does not alter the value of the potentials, we shall simply work with the multipole expansions presented in Eqs. (2.4) and (2.5).

\section{SCALAR WAVE EQUATION AND SOLUTION BY GREEN'S IDENTITY}

We consider a scalar field $\Psi$ that satisfies the wave equation

$$
\partial_{\alpha}\left(\sqrt{-g} g^{\alpha \beta} \partial_{\beta} \Psi\right)=0,
$$

where $g$ is the determinant of the metric. On a spacelike hypersurface $\Sigma$ we specify initial values for the field and its normal derivative. The field's behavior to the future of $\Sigma$ is then completely determined, and $\Psi$ can be expressed as $[48]$

$$
\begin{aligned}
\Psi(x)=\frac{1}{4 \pi} \int_{\Sigma}\left[G\left(x, x^{\prime}\right) \partial_{\alpha^{\prime}} \Psi\left(x^{\prime}\right)\right. \\
\left.-\Psi\left(x^{\prime}\right) \partial_{\alpha^{\prime}} G\left(x, x^{\prime}\right)\right] n^{\alpha^{\prime}} d V^{\prime}
\end{aligned}
$$

this is Green's identity in curved spacetime. Here, $x$ denotes a spacetime point to the future of $\Sigma$, and $x^{\prime}$ is a point on the hypersurface, which has $n^{\alpha^{\prime}}$ as its unit normal vector and $d V^{\prime}$ as its natural (invariant) volume element. The quantity $G\left(x, x^{\prime}\right)$ appearing inside the integral is the retarded Green's function for the wave equation of Eq. (3.1); this satisfies

$$
\partial_{\alpha}\left(\sqrt{-g} g^{\alpha \beta} \partial_{\beta} G\right)=-4 \pi \delta_{4}\left(x-x^{\prime}\right)
$$


together with a condition that $G\left(x, x^{\prime}\right)$ vanish if $x$ is in the causal past of $x^{\prime}$; here $\delta_{4}\left(x-x^{\prime}\right)$ denotes a fourdimensional Dirac distribution.

For the spacetime of Eq. (2.1), Eq. (3.1) takes the explicit form

$$
\left(\square+4 \Phi \partial_{t t}+8 A^{a} \partial_{t a}\right) \Psi=0,
$$

where $\square=-(\partial / \partial t)^{2}+\nabla^{2}$ is the flat-spacetime wave operator, Eq. (3.3) becomes

$$
\left(\square+4 \Phi \partial_{t t}+8 A^{a} \partial_{t a}\right) G=-4 \pi \delta_{4}\left(x-x^{\prime}\right),
$$

and Eq. (3.2) reduces to

$$
\begin{aligned}
\Psi(t, \boldsymbol{x})= & \frac{1}{4 \pi} \int\left[G\left(t, \boldsymbol{x} ; 0, \boldsymbol{x}^{\prime}\right) \dot{\Psi}\left(0, \boldsymbol{x}^{\prime}\right)-\Psi\left(0, \boldsymbol{x}^{\prime}\right) \partial_{t^{\prime}} G\left(t, \boldsymbol{x} ; 0, \boldsymbol{x}^{\prime}\right)\right]\left[1-4 \Phi\left(\boldsymbol{x}^{\prime}\right)\right] d^{3} x^{\prime} \\
& -\frac{1}{\pi} \int\left[G\left(t, \boldsymbol{x} ; 0, \boldsymbol{x}^{\prime}\right) \partial_{a^{\prime}} \Psi\left(0, \boldsymbol{x}^{\prime}\right)-\Psi\left(0, \boldsymbol{x}^{\prime}\right) \partial_{a^{\prime}} G\left(t, \boldsymbol{x} ; 0, \boldsymbol{x}^{\prime}\right)\right] A^{a}\left(\boldsymbol{x}^{\prime}\right) d^{3} x^{\prime},
\end{aligned}
$$

where $\Psi\left(0, \boldsymbol{x}^{\prime}\right)$ is the field's initial value on the hypersurface $t=0$, and $\dot{\Psi}\left(0, \boldsymbol{x}^{\prime}\right)$ the initial value for the field's time derivative.

Equation (3.5) can be solved perturbatively about flat spacetime. This method of computation originates from Ref. [43], and the particular implementation used here comes from Ref. [47]; a more detailed exposition can be found in Ref. [44]. We write

$$
G\left(x, x^{\prime}\right)=G^{\text {flat }}\left(x, x^{\prime}\right)+G^{1}\left(x, x^{\prime}\right)+\cdots,
$$

where

$$
G^{\text {flat }}\left(x, x^{\prime}\right)=\frac{\delta\left(t-t^{\prime}-\left|\boldsymbol{x}-\boldsymbol{x}^{\prime}\right|\right)}{\left|\boldsymbol{x}-\boldsymbol{x}^{\prime}\right|}
$$

is the retarded Green's function of the flat-spacetime wave equation, $\square G^{\text {flat }}=-4 \pi \delta_{4}\left(x-x^{\prime}\right), G^{1}\left(x, x^{\prime}\right)$ is a correction of order $\Phi$ and $\boldsymbol{A}$, and the "..." terms represent corrections of higher order in the gravitational potentials. Substituting Eq. (3.8) into Eq. (3.5) reveals that the first-order correction to the Green's function satisfies

$$
\square G^{1}\left(x, x^{\prime}\right)=-4\left(\Phi \partial_{t t}+2 A^{a} \partial_{t a}\right) G^{\mathrm{flat}}\left(x, x^{\prime}\right),
$$

in which the gravitational potentials are evaluated at $x$. The integration of this equation will be carried out in Sec. IV.

The Green's function of Eq. (3.7) can be decomposed into two parts. The first, which is dominated by $G^{\text {flat }}\left(x, x^{\prime}\right)$, is supported entirely on the past null cone of the field point $x$; this is the "direct" part of the Green's function [48, 49], which describes the direct propagation - at the speed of light — of a disturbance from $x^{\prime}$ to $x$. The second, which is contained in $G^{1}\left(x, x^{\prime}\right)$, is supported inside the past null cone of the field point $x$; this is the "tail" part of the Green's function [48, 49], which describes the delayed propagation — at speeds smaller than the speed of light - of a disturbance from $x$ to $x^{\prime}$. This delay is a curved-spacetime phenomenon: it can heuristically be understood as arising from a scattering of the radiation by the spacetime curvature. Note that
$G^{1}\left(x, x^{\prime}\right)$ contains both a direct part and a tail part; in the sequel we shall be interested only in its tail part, which we denote $G^{\text {tail }}\left(x, x^{\prime}\right)$.

Likewise, the scalar field of Eq. (3.6) can be decomposed into direct and tail parts. The direct part of the field represents the passage of the main wavefront, that is, the arrival at the observation point of that part of the initial wave packet that has propagated at the speed of light. The tail part of the field is what arrives after the main wavefront, delayed by some interaction with the spacetime curvature. In the sequel we shall be interested only in the tail part of the scalar field, that is, the field's behavior after the passage of the main wavefront; it is this behavior that reveals the influence of the spacetime curvature on the radiation. Ignoring terms that are quadratic in the gravitational potentials, we find from Eq. (3.6) that the tail part of the field is given by

$$
\begin{aligned}
\Psi^{\text {tail }}(t, \boldsymbol{x})=\frac{1}{4 \pi} \int & {\left[G^{\text {tail }}\left(t, \boldsymbol{x} ; 0, \boldsymbol{x}^{\prime}\right) \dot{\Psi}\left(0, \boldsymbol{x}^{\prime}\right)\right.} \\
& \left.-\Psi\left(0, \boldsymbol{x}^{\prime}\right) \partial_{t^{\prime}} G^{\text {tail }}\left(t, \boldsymbol{x} ; 0, \boldsymbol{x}^{\prime}\right)\right] d^{3} x^{\prime}
\end{aligned}
$$

where, as was noted before, $G^{\text {tail }}\left(x, x^{\prime}\right)$ is the tail part of $G^{1}\left(x, x^{\prime}\right)$.

\section{EVALUATION OF THE GREEN'S FUNCTION}

Equation (3.9) has the form of a wave equation with a source term, and its solution is $[43,44,47]$

$$
\begin{aligned}
G^{1}\left(x, x^{\prime}\right)= & \frac{1}{\pi} \int G^{\mathrm{flat}}\left(x, x^{\prime \prime}\right)\left(\Phi \partial_{t^{\prime \prime} t^{\prime \prime}}\right. \\
& \left.+2 A^{a} \partial_{t^{\prime \prime} a^{\prime \prime}}\right) G^{\mathrm{flat}}\left(x^{\prime \prime}, x^{\prime}\right) d^{4} x^{\prime \prime},
\end{aligned}
$$

where the gravitational potentials are evaluated at $x^{\prime \prime}$. Because the flat-spacetime Green's function $G^{\text {flat }}\left(x^{\prime \prime}, x^{\prime}\right)$, given explicitly by Eq. (3.8), depends only on the difference between $x^{\prime \prime}$ and $x^{\prime}$, the derivatives with respect to 
$x^{\prime \prime}$ can be converted (by introducing a minus sign) into derivatives with respect to $x^{\prime}$, and these can be taken outside the integral. The first-order correction to the Green's function can thus be expressed as

$$
G^{1}\left(x, x^{\prime}\right)=2 \partial_{t^{\prime} t^{\prime}} P\left(x, x^{\prime}\right)+4 \partial_{t^{\prime} a^{\prime}} P^{a}\left(x, x^{\prime}\right),
$$

in terms of two-point functions defined by

$$
\begin{aligned}
P\left(x, x^{\prime}\right) & =\frac{1}{2 \pi} \int G^{\mathrm{flat}}\left(x, x^{\prime \prime}\right) \Phi\left(\boldsymbol{x}^{\prime \prime}\right) G^{\mathrm{flat}}\left(x^{\prime \prime}, x^{\prime}\right) d^{4} x^{\prime \prime} \\
& \equiv\langle\Phi\rangle
\end{aligned}
$$

and

$$
\begin{aligned}
P^{a}\left(x, x^{\prime}\right) & =\frac{1}{2 \pi} \int G^{\mathrm{flat}}\left(x, x^{\prime \prime}\right) A^{a}\left(\boldsymbol{x}^{\prime \prime}\right) G^{\mathrm{flat}}\left(x^{\prime \prime}, x^{\prime}\right) d^{4} x^{\prime \prime} \\
& \equiv\left\langle A^{a}\right\rangle .
\end{aligned}
$$

In these integrations, the product of the flat-spacetime Green's functions is supported on the two-dimensional surface $\mathcal{S}$ formed by the intersection of $x$ 's past light cone and $x^{\prime}$ 's future light cone. The two-point functions are therefore the average of the gravitational potentials over $\mathcal{S}$, and we have introduced this notation explicitly in Eqs. (4.2) and (4.3). We have indicated that the potentials are functions of the spatial variables $\boldsymbol{x}^{\prime \prime}$ only; they do not depend on the time variable $t^{\prime \prime}$.

It is easy to see that $\mathcal{S}$ will have a large area if $x$ and $x^{\prime}$ are widely separated in time. In these circumstances, the spatial vector $\boldsymbol{x}^{\prime \prime}$ that appears in Eqs. (4.2) and (4.3) will be large, and the gravitational potentials will be adequately represented by the multipole expansions of Eqs. (2.4) and (2.5). More precisely stated, in Appendix A we prove that if the time delay $t-t^{\prime}$ satisfies the condition

$$
t-t^{\prime}>r+3 r^{\prime}+2 \mathcal{R},
$$

where $r \equiv|\boldsymbol{x}|, r^{\prime} \equiv\left|\boldsymbol{x}^{\prime}\right|$, and $\mathcal{R}$ is the maximum radius of the matter distribution, then $r^{\prime \prime} \equiv\left|\boldsymbol{x}^{\prime \prime}\right|>\mathcal{R}$ and $\Phi\left(\boldsymbol{x}^{\prime \prime}\right)$ and $A^{a}\left(\boldsymbol{x}^{\prime \prime}\right)$ can both be expressed as a multipole expansion in powers of $1 / r^{\prime \prime}$. For the remainder of the paper we will assume that Eq. (4.4) holds. This means that the time $t$ that appears in Eq. (3.10) will be restricted by $t>r+3 \mathcal{L}+2 \mathcal{R}$, where $r$ is the distance to the observation point, $\mathcal{L} \equiv \max \left(r^{\prime}\right)$ the radius of the region that contains the field's initial data, and $\mathcal{R}$ the radius of the region occupied by the matter. Notice that we are explicitly assuming that the field's initial configuration is compactly supported: the field's initial values are assumed to be zero outside of a sphere of radius $\mathcal{L}$.

Taking into account Eq. (4.4), we substitute the multipole expansions of Eqs. (2.4) and (2.5) into Eqs. (4.2) and (4.3). Introducing the sequence of two-point functions

$$
\begin{aligned}
U\left(x, x^{\prime}\right) & =\frac{1}{2 \pi} \int G^{\mathrm{flat}}\left(x, x^{\prime \prime}\right) \frac{1}{r^{\prime \prime}} G^{\mathrm{flat}}\left(x^{\prime \prime}, x^{\prime}\right) d^{4} x^{\prime \prime} \\
& \equiv\left\langle r^{-1}\right\rangle \\
U_{a}\left(x, x^{\prime}\right) & =\left\langle\partial_{a} r^{-1}\right\rangle
\end{aligned}
$$

and generically,

$$
U_{N}\left(x, x^{\prime}\right)=\left\langle\partial_{N} r^{-1}\right\rangle,
$$

we find that Eqs. (4.2) and (4.3) become

$$
P\left(x, x^{\prime}\right)=-M U\left(x, x^{\prime}\right)-\sum_{n=1}^{\infty} \frac{(-1)^{n}}{n !} M^{N} U_{N}\left(x, x^{\prime}\right)
$$

and

$$
P^{a}\left(x, x^{\prime}\right)=J^{a b} U_{b}\left(x, x^{\prime}\right)-\sum_{n=2}^{\infty} \frac{(-1)^{n}}{n !} J^{a N} U_{N}\left(x, x^{\prime}\right) .
$$

We see from Eqs. (4.1), (4.8), and (4.9) that $G^{1}\left(x, x^{\prime}\right)$ can be constructed from the infinite sequence of two-point functions $U_{N}\left(x, x^{\prime}\right)$. Our next task is to compute these.

Suppose that we already have $U_{N}\left(x, x^{\prime}\right)$, and that we want $U_{N a}$, the next member of the sequence. In schematic notation we have $U_{N}=\int G f G d x^{\prime \prime}$ and $U_{N a}=$ $\int G\left(\partial_{a^{\prime \prime}} f\right) G d x^{\prime \prime}$, where $f \equiv \partial_{N^{\prime \prime}}\left(r^{\prime \prime-1}\right)$. By letting $\partial_{a^{\prime \prime}}$ act on the second Green's function we rewrite this as $U_{N a}=\int G \partial_{a^{\prime \prime}}(f G) d x^{\prime \prime}+\partial_{a^{\prime}} \int G f G d x^{\prime \prime}$, where we have used the fact that the second Green's function depends only on $x^{\prime \prime}-x^{\prime}$. The first integral is then integrated by parts, and if we use the fact that the first Green's function depends only on $x-x^{\prime \prime}$, we obtain $U_{N a}=\partial_{a} \int G f G d x^{\prime \prime}+\partial_{a^{\prime}} \int G f G d x^{\prime \prime}$. The sequence therefore obeys the recursion relation

$$
U_{N a}\left(x, x^{\prime}\right)=\left(\partial_{a}+\partial_{a^{\prime}}\right) U_{N}\left(x, x^{\prime}\right),
$$

and each member can be obtained from $U\left(x, x^{\prime}\right)$ by repeated application of the symmetric derivative operator $\partial_{a}+\partial_{a^{\prime}}$. Explicitly,

$$
\begin{aligned}
U_{a}\left(x, x^{\prime}\right) & =\left(\partial_{a}+\partial_{a^{\prime}}\right) U\left(x, x^{\prime}\right) \\
U_{N}\left(x, x^{\prime}\right) & =\left(\partial_{a_{1}}+\partial_{a_{1}^{\prime}}\right) \cdots\left(\partial_{a_{n}}+\partial_{a_{n}^{\prime}}\right) U\left(x, x^{\prime}\right),
\end{aligned}
$$

and we see that the key to obtaining $G^{1}\left(x, x^{\prime}\right)$ is to compute the two-point function $U\left(x, x^{\prime}\right)$ defined by Eq. (4.5).

An almost identical set of manipulations would reveal that the two-point functions depend only on $t-t^{\prime}$, since $\left(\partial_{t}+\partial_{t^{\prime}}\right) U_{N}\left(x, x^{\prime}\right)=0$. This property extends to $P\left(x, x^{\prime}\right)$ and $P^{a}\left(x, x^{\prime}\right)$, as follows directly from Eqs. (4.2), (4.3) and the fact that the gravitational potentials do not depend on time. Finally, Eq. (4.1) implies that $G^{1}\left(x, x^{\prime}\right)$ also depends only on $t-t^{\prime}$; this conclusion follows directly from the stationary property of the spacetime.

We must now evaluate $U\left(x, x^{\prime}\right)$. In Appendix A we show that for $t-t^{\prime}>r+r^{\prime}$ - which is implied by Eq. (4.4) - we have [43, 44, 47]

$$
U\left(x, x^{\prime}\right)=\frac{1}{R} \ln \frac{t-t^{\prime}+R}{t-t^{\prime}-R},
$$

where $R \equiv\left|\boldsymbol{x}-\boldsymbol{x}^{\prime}\right|$ is the Euclidean distance between $\boldsymbol{x}$ and $\boldsymbol{x}^{\prime}$. We see that the spatial dependence of $U\left(x, x^{\prime}\right)$ is contained entirely in $R$, which is antisymmetric in $\boldsymbol{x}$ and 
$\boldsymbol{x}^{\prime}$. Thus $\left(\partial_{a}+\partial_{a^{\prime}}\right) R=0$, and from this it follows that $U_{a}\left(x, x^{\prime}\right)$ and all other members of the sequence vanish for $t-t^{\prime}>r+r^{\prime}$. Then Eqs. (4.8) and (4.9) imply $P\left(x, x^{\prime}\right)=-M U\left(x, x^{\prime}\right), P^{a}\left(x, x^{\prime}\right)=0$, and Eq. (4.1) gives

$$
G^{1}\left(x, x^{\prime}\right)=-\frac{8 M\left(t-t^{\prime}\right)}{\left[\left(t-t^{\prime}\right)^{2}-R^{2}\right]^{2}} .
$$

This result is valid under the condition stated in Eq. (4.4). For $t>t^{\prime}+r+3 r^{\prime}+2 \mathcal{R}$, therefore, the full scalar Green's function $G\left(x, x^{\prime}\right)$ is given by the expression of Eq. (4.14), except for corrections of higher order in the gravitational potentials. This result is remarkable because it shows that the late-time behavior of the Green's function is governed entirely by the spherical, $1 / r$ part of the gravitational potentials. In Appendix A we prove that $U_{a}\left(x, x^{\prime}\right)$ and other members of the sequence are nonzero when $t-t^{\prime}<r+r^{\prime}$. This inequality is incompatible with Eq. (4.4), which ensures that the gravitational potentials can safely be expressed as multipole expansions. In other words, $U_{a}\left(x, x^{\prime}\right)$ and other members of the sequence are nonzero only where they not properly defined.

\section{LATE-TIME BEHAVIOR OF THE FIELD}

In this section we substitute Eq. (4.14) into Eq. (3.10) and compute the scalar field $\Psi(t, \boldsymbol{x})$. We shall represent the field point $\boldsymbol{x}$ in terms of spherical coordinates $(r, \theta, \phi)$, and the source point $\boldsymbol{x}^{\prime}$ in terms of $\left(r^{\prime}, \theta^{\prime}, \phi^{\prime}\right)$. We shall use the short-hand notation $\Omega=(\theta, \phi)$ and $\Omega^{\prime}=\left(\theta^{\prime}, \phi^{\prime}\right)$.

We need to specify initial conditions for the field and its time derivative. We make the specific choices

$$
\Psi\left(0, \boldsymbol{x}^{\prime}\right)=C\left(r^{\prime}\right) Y_{l}^{m}\left(\Omega^{\prime}\right), \quad \dot{\Psi}\left(0, \boldsymbol{x}^{\prime}\right)=\dot{C}\left(r^{\prime}\right) Y_{l}^{m}\left(\Omega^{\prime}\right),
$$

where $Y_{l}^{m}\left(\Omega^{\prime}\right)$ are spherical-harmonic functions, and $C\left(r^{\prime}\right)$ and $\dot{C}\left(r^{\prime}\right)$ are two arbitrary functions supported in the interval $0<r^{\prime}<\mathcal{L}<\infty$. We therefore suppose that $\Psi$ is initially a pure multipole field of degree $l$ and azimuthal index $m$ (assumed for simplicity to be the same for both $\Psi$ and $\dot{\Psi})$, and that the initial data is confined to a ball of radius $\mathcal{L}$. Note that $C\left(r^{\prime}\right)$ and $\dot{C}\left(r^{\prime}\right)$ are two distinct and unrelated functions of $r^{\prime}$; the overdot simply means that $\dot{C}\left(r^{\prime}\right)$ specifies the initial data for the field's time derivative, and it should not suggest that $\dot{C}\left(r^{\prime}\right)$ is the time derivative of $C\left(r^{\prime}\right)$. Below we will need moments of these functions, $C_{l}$ and $\dot{C}_{l}$, defined by

$$
C_{l} \equiv \int C\left(r^{\prime}\right)\left(r^{\prime}\right)^{l+2} d r^{\prime}
$$

and a very similar relation holding for $\dot{C}_{l}$. Notice that as defined here, $C_{l}$ is the integral of $C\left(r^{\prime}\right)$ multiplied by $\left(r^{\prime}\right)^{l+2}$, not $\left(r^{\prime}\right)^{l}$; the two additional powers of $r^{\prime}$ are contributed by the volume element in spherical coordinates: $d^{3} x^{\prime}=r^{\prime 2} d r^{\prime} d \Omega^{\prime}$, where $d \Omega^{\prime}=\sin \theta^{\prime} d \theta^{\prime} d \phi^{\prime}$.
We consider the behavior of the scalar field at times $t>$ $r+3 \mathcal{L}+2 \mathcal{R}$, as demanded by the inequality of Eq. (4.4). Substituting Eq. (4.14) into Eq. (3.10) we obtain

$$
\Psi^{>}(t, \boldsymbol{x})=\dot{S}(t, \boldsymbol{x})+\frac{\partial}{\partial t} S(t, \boldsymbol{x}),
$$

where the superscript ">" indicates that Eq. (4.4) is being enforced, and where

$$
S(t, \boldsymbol{x}) \equiv \frac{1}{4 \pi} \int G^{1}\left(t, \boldsymbol{x} ; 0, \boldsymbol{x}^{\prime}\right) C\left(r^{\prime}\right) Y_{l}^{m}\left(\Omega^{\prime}\right) d^{3} x^{\prime},
$$

with a very similar relation defining $\dot{S}(t, \boldsymbol{x})$. Here the Green's function $G^{1}\left(t, \boldsymbol{x} ; 0, \boldsymbol{x}^{\prime}\right)$ is given by Eq. (4.14), and the overdot on $\dot{S}$ means that this quantity is constructed from $\dot{C}$ in the same way that $S$ is constructed from $C$; it does not mean that $\dot{S}$ is the time derivative of $S$. To compute $S(t, \boldsymbol{x})$ and $\dot{S}(t, \boldsymbol{x})$ we shall first carry out the angular integrations to obtain

$$
Q\left(t, \boldsymbol{x} ; r^{\prime}\right) \equiv \frac{1}{4 \pi} \int G^{1}\left(t, \boldsymbol{x} ; 0, \boldsymbol{x}^{\prime}\right) Y_{l}^{m}\left(\Omega^{\prime}\right) d \Omega^{\prime},
$$

and then perform the remaining radial integration:

$$
S(t, \boldsymbol{x})=\int Q\left(t, \boldsymbol{x} ; r^{\prime}\right) C\left(r^{\prime}\right) r^{\prime 2} d r^{\prime},
$$

with a very similar relation holding for $\dot{S}(t, \boldsymbol{x})$.

The Green's function of Eq. (4.14) can be expressed as

$$
G^{1}\left(t, \boldsymbol{x} ; 0, \boldsymbol{x}^{\prime}\right)=-\frac{8 M t}{\left(t^{2}-r^{2}+2 r r^{\prime} \cos \gamma-r^{\prime 2}\right)^{2}},
$$

where

$$
\cos \gamma=\cos \theta \cos \theta^{\prime}+\sin \theta \sin \theta^{\prime} \cos \left(\phi-\phi^{\prime}\right)
$$

gives the angle between $\boldsymbol{x}$ and $\boldsymbol{x}^{\prime}$. We use the binomial theorem to rewrite Eq. (5.7) as

$$
\begin{aligned}
G^{1}= & -\frac{8 M t}{\left(t^{2}-r^{2}-r^{\prime 2}\right)^{2}} \sum_{p=0}^{\infty}(-1)^{p}(p+1) \\
& \times\left(\frac{2 r r^{\prime}}{t^{2}-r^{2}-r^{\prime 2}}\right)^{p}(\cos \gamma)^{p}
\end{aligned}
$$

and substitution into Eq. (5.5) gives

$$
\begin{aligned}
Q\left(t, \boldsymbol{x} ; r^{\prime}\right)= & -\frac{8 M t}{\left(t^{2}-r^{2}-r^{\prime 2}\right)^{2}} \sum_{p=0}^{\infty}(-1)^{p}(p+1) \\
& \times\left(\frac{2 r r^{\prime}}{t^{2}-r^{2}-r^{\prime 2}}\right)^{p} I_{p}(\Omega),
\end{aligned}
$$

where

$$
I_{p}(\Omega) \equiv \frac{1}{4 \pi} \int(\cos \gamma)^{p} Y_{l}^{m}\left(\Omega^{\prime}\right) d \Omega^{\prime} .
$$


These angular integrations are carried out in Appendix $\mathrm{B}$, where we show that

$$
I_{p}(\Omega)=\frac{2^{l}(l+2 n) !(l+n) !}{(2 l+2 n+1) ! n !} Y_{l}^{m}(\Omega)
$$

if $p=l+2 n$, where $n=0,1,2, \cdots$; for all other values of $p$, and in particular for $p<l$, the angular functions vanish. The infinite sum in Eq. (5.9) therefore starts at $p=l$, and it contains even terms only if $l$ is even, or odd terms only if $l$ is odd. After some simple algebra, substitution of Eq. (5.11) into Eq. (5.9) yields

$$
Q\left(t, \boldsymbol{x} ; r^{\prime}\right)=4 M(-1)^{l+1} \frac{(2 l+2) ! !}{(2 l+1) ! !} \frac{\left(r r^{\prime}\right)^{l} t}{\left(t^{2}-r^{2}-r^{\prime 2}\right)^{l+2}} Y_{l}^{m}(\Omega) \sum_{n=0}^{\infty} \frac{(l+1+2 n) !}{(l+1) !} \frac{(l+n) !}{l ! n !} \frac{(2 l+1) !}{(2 l+1+2 n) !}\left(\frac{2 r r^{\prime}}{t^{2}-r^{2}-r^{\prime 2}}\right)^{2 n}
$$

which we rewrite as

$$
\begin{aligned}
Q\left(t, \boldsymbol{x} ; r^{\prime}\right)= & 4 M(-1)^{l+1} \frac{(2 l+2) ! !}{(2 l+1) ! !} \frac{\left(r r^{\prime}\right)^{l} t}{\left(t^{2}-r^{2}\right)^{l+2}} Y_{l}^{m}(\Omega) \\
& \times\left(1+\frac{r^{\prime 2}}{t^{2}-r^{2}-r^{\prime 2}}\right)^{l+2} \sum_{n=0}^{\infty} \frac{(l+1+2 n) !}{(l+1) !} \frac{(l+n) !}{l ! n !} \frac{(2 l+1) !}{(2 l+1+2 n) !}\left(\frac{2 r r^{\prime}}{t^{2}-r^{2}-r^{\prime 2}}\right)^{2 n} .
\end{aligned}
$$

In Eq. (5.12), the essential information appears on the first line, and the second line (which includes the infinite sum) contributes only an overall factor of $1+\varepsilon_{1}+\varepsilon_{2}^{2}$, with $\varepsilon_{1}$ denoting a small quantity of order $r^{\prime 2} /\left(t^{2}-r^{2}-r^{\prime 2}\right)$ and $\varepsilon_{2}$ one of order $r r^{\prime} /\left(t^{2}-r^{2}-r^{\prime 2}\right)$. Because $r^{\prime}$ is itself of order $\mathcal{L}$, we have that

$$
\varepsilon_{1}=O\left(\frac{\mathcal{L}^{2}}{t^{2}-r^{2}-\mathcal{L}^{2}}\right), \quad \varepsilon_{2}=O\left(\frac{r \mathcal{L}}{t^{2}-r^{2}-\mathcal{L}^{2}}\right)
$$

These small corrections to the leading factor of Eq. (5.12) will be of no concern to us.

Substitution of Eq. (5.12) into Eq. (5.6) gives

$$
\begin{aligned}
S(t, \boldsymbol{x})= & 4 M(-1)^{l+1} \frac{(2 l+2) ! !}{(2 l+1) ! !} C_{l} \frac{r^{l} t}{\left(t^{2}-r^{2}\right)^{l+2}} Y_{l}^{m}(\Omega) \\
& \times\left[1+O\left(\varepsilon_{1}\right)+O\left(\varepsilon_{2}^{2}\right)\right],
\end{aligned}
$$

and the same relation also gives $\dot{S}(t, \boldsymbol{x})$ if $\dot{C}_{l}$ is substituted in place of $C_{l}$. These results can now be substituted into Eq. (5.3), and this yields

$$
\begin{aligned}
\Psi^{>}(t, r, \Omega)= & 4 M(-1)^{l+1} \frac{(2 l+2) ! !}{(2 l+1) ! !} \frac{r^{l} t}{\left(t^{2}-r^{2}\right)^{l+2}} Y_{l}^{m}(\Omega) \\
& \times\left\{\dot{C}_{l}+\left[1-2(l+2) \frac{t^{2}}{t^{2}-r^{2}}\right] \frac{C_{l}}{t}\right\} \\
& \times\left[1+O\left(\varepsilon_{1}\right)+O\left(\varepsilon_{2}{ }^{2}\right)\right] .
\end{aligned}
$$

This expression is valid for $t>r+3 \mathcal{L}+2 \mathcal{R}$, in which $\mathcal{R}$ marks the boundary of the matter distribution, and $\mathcal{L}$ the extension of the field's initial configuration. Equation (5.15) shows that generically, the field behaves as $r^{l} t /\left(t^{2}-r^{2}\right)^{l+2}$, but that its behavior goes to $r^{l} /\left(t^{2}-\right.$ $\left.r^{2}\right)^{l+2}$ instead if $\dot{\Psi}\left(0, \boldsymbol{x}^{\prime}\right)=0$, that is, if the initial data is time symmetric.

If $t \gg r$ the general result of Eq. (5.15) takes the simpler form

$$
\begin{aligned}
\Psi(t \gg r, r, \Omega)= & 4 M(-1)^{l+1} \frac{(2 l+2) ! !}{(2 l+1) ! !} \frac{r^{l}}{t^{2 l+3}} Y_{l}^{m}(\Omega) \\
& \times\left(\dot{C}_{l}-\frac{2 l+3}{t} C_{l}\right),
\end{aligned}
$$

which shows that in this limit, the field behaves as $r^{l} / t^{2 l+3}$. This is the behavior of the scalar field near future timelike infinity. Equation (5.16) is valid up to a fractional correction of order $r^{2} / t^{2}$.

To obtain the limiting behavior of the field at future null infinity, we introduce the retarded time coordinate $u \equiv t-r$ and the advanced time coordinate $v \equiv t+r$. We then take the limit of $r \Psi^{>}$as $v \rightarrow \infty$, which gives

$$
\begin{aligned}
r \Psi(v=\infty, u, \Omega)= & 2 M(-1)^{l+1} \frac{(l+1) !}{(2 l+1) ! !} \frac{1}{u^{l+2}} Y_{l}^{m}(\Omega) \\
& \times\left(\dot{C}_{l}-\frac{l+2}{u} C_{l}\right) .
\end{aligned}
$$

At future null infinity, therefore, $r \Psi$ behaves as $1 / u^{l+2}$. Equation (5.17) is valid up to a fractional correction of order $\mathcal{L}^{2} / u^{2}$.

Equation (5.15), and its two limiting cases (5.16) and (5.17), give us our new generalized formulation of Price's falloff theorem for a scalar field $\Psi$ starting with the initial data of Eq. (5.1) and propagating in the spacetime of Eq. (2.1). It states that for $t>r+3 \mathcal{L}+2 \mathcal{R}$, the field behaves as it would in a spherically symmetric spacetime of the same mass $M$; deviations from spherical symmetry do not affect the field at such late times. 
It has long been known that the radiative decay of a scalar field in an asymptotically-flat spacetime goes from a $t^{-(2 l+3)}$ behavior near future timelike infinity to a $u^{-(l+2)}$ behavior at future null infinity. Our derivation offers a very clear explanation for this transition: The two different behaviors arise naturally by taking different limits of the more general description $r \Psi \propto$ $r^{l+1} t /\left(t^{2}-r^{2}\right)^{l+2}=(1-u / v)^{l+1}(1+u / v) /\left(2^{l+2} u^{l+2}\right)$.

\section{RADIATIVE FALLOFF IN SPHEROIDAL COORDINATES}

In the preceding sections we found that the late-time behavior of a scalar field in a weakly curved spacetime is governed entirely by the spherically-symmetric part of the scalar potential $\Phi$, and that it is oblivious to the vector potential $\boldsymbol{A}$ and the nonspherical part of $\Phi$. In particular, we found that the spacetime's total angular momentum plays no role in the description of the radiative decay.

A very different conclusion seems to emerge from previous studies of the late-time dynamics of a scalar field in the spacetime of a Kerr black hole [33-41]. These reveal a much more complicated picture in which an initial $Y_{l}^{m}$ mode gets converted into modes of lower degree. As a result of mode conversion, $\Psi(t \gg r)$ behaves as $1 / t^{2 l+3}$ if $l=m$ or $l=m+1$, as $1 / t^{l+m+1}$ if $l \geq m+2$ and $l-m$ is even, or as $1 / t^{l+m+2}$ if $l \geq m+2$ and $l-m$ is odd - see Eq. (1.7). Furthermore, $r \Psi(v=\infty)$ behaves as $1 / u^{l+2}$ if $l=m$ or $l=m+1$, or as $1 / u^{l}$ if $l \geq m+2-$ see Eq. (1.8). According to Hod [37], who derived these results for the first time, the difference in the field's falloff must be attributed to the angular momentum of the black hole, but this interpretation is in direct conflict with our previous conclusion.

In this section we show that the results reviewed in the preceding paragraph are largely an artifact of the Boyer-Lindquist coordinates used to represent the Kerr metric. We show that the mode conversion has nothing to do with the spacetime's angular momentum, and that we can reproduce all of the preceding results within the approach developed in this paper. There is therefore no contradiction between the statements made in Sec. V and the results derived by Hod [37].

It is known that in the limit $M \rightarrow 0$ (where $M$ denotes the mass of the black hole), the Kerr metric reduces to the metric of flat spacetime in spheroidal coordinates $(r, \theta, \phi)$. These are related to a Cartesian frame $(x, y, z)$ by the relations

$$
x=\rho \sin \theta \cos \phi, \quad y=\rho \sin \theta \sin \phi, \quad z=r \cos \theta,
$$

where $\rho \equiv \sqrt{r^{2}+e^{2}}$, with $e$ denoting the ellipticity of the coordinate system (the angular momentum of a Kerr black hole is equal to $M e$ ). The weak-field limit of the Kerr metric is then naturally represented as a deforma- tion of the Minkowski metric expressed in spheroidal coordinates. This suggests that a meaningful comparison of results must involve a translation of the computations carried out in Sec. V into this coordinate system. [Notice that while we use here the notation $(r, \theta, \phi)$ for the spheroidal coordinates, these are in fact distinct from the spherical coordinates used in Sec. V; we have discarded the distinguishing carets used in Sec. I to keep the notation simple.]

This is what we shall do in this Section. The fact that the coordinates of Eq. (6.1) are not spherical — even though they become increasingly spherical as $r$ grows much larger than $e$ - will be seen to be crucially important. We will show that the difference in the coordinate systems, which is measured by the parameter $e$, is at the origin of the difference between the two descriptions of the falloff behavior. While the description is very simple in the original spherical coordinates (because the late-time Green's function is insensitive to deviations from spherical symmetry), it is much more complicated in the spheroidal coordinates (because they obscure the underlying spherical symmetry of the problem). The two descriptions, of course, are fully compatible.

We shall retrace the steps taken in Sec. V and translate these computations into the spheroidal coordinates of Eq. (6.1). We suppose once more that the field's initial configuration is a pure multipole field of degree $l$ and azimuthal index $m$; we describe it, in the spheroidal coordinates, by Eq. (5.1). The late-time behavior of the field is once more given by Eqs. (5.3) and (5.4), in which the Green's function can be substituted from Eq. (4.14). The volume element is now $d^{3} x^{\prime}=\left(r^{2}+e^{2} \cos ^{2} \theta^{\prime}\right) d r^{\prime} d \Omega^{\prime}$, and the quantity $R^{2}$ that appears in the Green's function is now given by $R^{2}=\rho^{2}+\rho^{\prime 2}-e^{2} \cos ^{2} \theta-$ $2 \rho \rho^{\prime} \sin \theta \sin \theta^{\prime} \cos \left(\phi-\phi^{\prime}\right)-2 r r^{\prime} \cos \theta \cos \theta^{\prime}-e^{2} \cos ^{2} \theta^{\prime}$. The appropriate replacement for Eq. (5.5) is then

$$
\begin{aligned}
Q\left(t, \boldsymbol{x} ; r^{\prime}\right)= & \frac{1}{4 \pi} \int G^{1}\left(t, \boldsymbol{x} ; 0, \boldsymbol{x}^{\prime}\right) Y_{l}^{m}\left(\Omega^{\prime}\right) \\
& \times\left(r^{\prime 2}+e^{2} \cos ^{2} \theta^{\prime}\right) d \Omega^{\prime},
\end{aligned}
$$

and Eq. (5.6) must be replaced by

$$
S(t, \boldsymbol{x})=\int Q\left(t, \boldsymbol{x} ; r^{\prime}\right) C\left(r^{\prime}\right) d r^{\prime}
$$

with a very similar relation holding for $\dot{S}(t, \boldsymbol{x})$. The scalar field is then given by Eq. (5.3), or $\Psi(t, \boldsymbol{x})=$ $\dot{S}(t, \boldsymbol{x})+\partial_{t} S(t, \boldsymbol{x})$. In the following we will go through a sketchy evaluation of $Q\left(t, \boldsymbol{x} ; r^{\prime}\right)$; this will be sufficient to show that our method, when combined with the spheroidal coordinates of Eq. (6.1), reproduces the Kerr results reviewed at the beginning of this section.

The Green's function of Eq. (4.14) takes the explicit form 


$$
G^{1}\left(t, \boldsymbol{x} ; 0, \boldsymbol{x}^{\prime}\right)=-\frac{8 M t}{T^{4}}\left[1+\frac{2 \rho \rho^{\prime}}{T^{2}} \sin \theta \sin \theta^{\prime} \cos \left(\phi-\phi^{\prime}\right)+\frac{2 r r^{\prime} \cos \theta}{T^{2}} \cos \theta^{\prime}+\frac{e^{2}}{T^{2}} \cos ^{2} \theta^{\prime}\right]^{-2}
$$

where

$$
T^{2} \equiv t^{2}-\rho^{2}-\rho^{\prime 2}+e^{2} \cos ^{2} \theta .
$$

Making repeated use of the binomial theorem, we re-express the Green's function as

$$
G^{1}\left(t, \boldsymbol{x} ; 0, \boldsymbol{x}^{\prime}\right)=-\frac{8 M t}{T^{4}} \sum_{p=0}^{\infty} \sum_{q=0}^{p} \sum_{n=0}^{q} \frac{(-1)^{p}(p+1) !}{(p-q) !(q-n) ! n !} \frac{e^{2 n}\left(2 r r^{\prime} \cos \theta\right)^{q-n}\left(2 \rho \rho^{\prime}\right)^{p-q}}{T^{2 p}} S^{p-q} C^{q+n},
$$

where $S \equiv \sin \theta \sin \theta^{\prime} \cos \left(\phi-\phi^{\prime}\right)$ and $C \equiv \cos \theta^{\prime}$. After substituting this into Eq. (6.2) and rearranging the sums, we obtain

$$
Q\left(t, \boldsymbol{x} ; r^{\prime}\right)=-\frac{8 M t}{T^{4}} \sum_{a=0}^{\infty} \sum_{b=0}^{\infty} \sum_{c=\{b / 2\}}^{b} \frac{(-1)^{a+c}(a+c+1) !}{a !(2 c-b) !(b-c) !} \frac{e^{2(b-c)}\left(2 r r^{\prime} \cos \theta\right)^{2 c-b}\left(2 \rho \rho^{\prime}\right)^{a}}{T^{2(a+c)}} I_{a b}\left(r^{\prime}, \Omega\right)
$$

where $\{b / 2\}$ stands for $b / 2$ if $b$ is even, or for $(b+1) / 2$ if $b$ is odd, and

$$
I_{a b}\left(r^{\prime}, \Omega\right) \equiv \frac{1}{4 \pi} \int\left(r^{\prime 2}+e^{2} C^{2}\right) S^{a} C^{b} Y_{l}^{m}\left(\Omega^{\prime}\right) d \Omega^{\prime}
$$

are angular integrations that generalize Eq. (5.10).

We evaluate the functions $I_{a b}\left(r^{\prime}, \Omega\right)$ in Appendix C. The most important task for our purposes is to determine the smallest values of $a$ and $b$ such that the functions do not vanish; we denote these $a^{*}$ and $b^{*}$, respectively. In Appendix $\mathrm{C}$ we show that $a^{*}=m$, and that $b^{*}=0$ if $l=m, b^{*}=1$ if $l=m+1$, and $b^{*}=l-m-2$ if $l \geq m+2$. In all cases we find that $I_{a^{*} b^{*}}\left(r^{\prime}, \Omega\right) \propto Y_{m}^{m}(\Omega)$.

We can now evaluate $Q\left(t, \boldsymbol{x} ; r^{\prime}\right)$ near future timelike infinity (for $t \gg r$ ). Assuming that $r$ and $r^{\prime}$ are both much larger than $e$, and that $r \gg r^{\prime}$, we use the approximations $\rho \simeq r, \rho^{\prime} \simeq r^{\prime}$, and $T^{2} \simeq t^{2}$. A typical term in the infinite sum of Eq. (6.7) is then proportional to $\left(r r^{\prime}\right)^{a-b+2 c}(\cos \theta)^{2 c-b} I_{a b} / t^{2(a+c)+3}$. The dominant term comes with $a=a^{*}, b=b^{*}$, and $c=\left\{b^{*} / 2\right\}$, and we find that

$$
Q(t \gg r) \propto \frac{\left(r r^{\prime}\right)^{m+\epsilon}}{t^{2 m+b^{*}+3+\epsilon}}(\cos \theta)^{\epsilon} Y_{m}^{m}(\Omega),
$$

where $\epsilon=0$ if $b^{*}$ is even, and $\epsilon=1$ if $b^{*}$ is odd. Using the previously determined values of $b^{*}$, we obtain the results displayed in Table I. Notice that when $l-m$ is even, the angular dependence is given by $Y_{m}^{m}(\Omega)$, the mode of lowest degree compatible with the fixed value of $m$; when $l-m$ is odd, we have instead $\cos \theta Y_{m}^{m}(\Omega) \propto Y_{m+1}^{m}(\Omega)$, which is the lowest accessible mode given that mode conversion must preserve the parity of the initial angular profile. These results are identical to those reviewed at the beginning of the section and displayed in Eq. (1.7).

Let us now examine the behavior of $r Q\left(t, \boldsymbol{x} ; r^{\prime}\right)$ at future null infinity (in the limit $v \equiv t+r \rightarrow \infty$ );
TABLE I: Behavior of $Q\left(t, \boldsymbol{x} ; r^{\prime}\right)$ near future timelike infinity.

\begin{tabular}{ll}
\hline \hline If $\ldots$ & $Q(t \gg r)$ is proportional to $\ldots$ \\
\hline$l=m$ & $\left(r r^{\prime}\right)^{m} Y_{m}^{m}(\Omega) / t^{2 m+3}$ \\
$l=m+1$ & $\left(r r^{\prime}\right)^{m+1} \cos \theta Y_{m}^{m}(\Omega) / t^{2 m+5}$ \\
$l \geq m+2, l-m$ even & $\left(r r^{\prime}\right)^{m} Y_{m}^{m}(\Omega) / t^{l+m+1}$ \\
$l \geq m+2, l-m$ odd & $\left(r r^{\prime}\right)^{m+1} \cos \theta Y_{m}^{m}(\Omega) / t^{l+m+2}$ \\
\hline \hline
\end{tabular}

we shall express this as a function of $u \equiv t-r$. Here we use the approximations $\rho \simeq r, \rho^{\prime} \simeq r^{\prime}$, and $T^{2} \simeq u v$. After multiplication by $r$, we find that a typical term in the infinite sum of Eq. (6.7) is proportional to $r t\left(r r^{\prime}\right)^{a-b+2 c}(\cos \theta)^{2 c-b} I_{a b} / T^{2(a+c)+4} \propto$ $\left(r^{\prime}\right)^{a-b+2 c}(\cos \theta)^{2 c-b} I_{a b} /\left(u^{a+c+2} v^{b-c}\right)$. In the limit $v \rightarrow$ $\infty$, the only surviving terms are those for which $c=b$. The dominant term is then

$$
Q(v=\infty) \propto \frac{\left(r^{\prime}\right)^{m+b^{*}}}{u^{m+b^{*}+2}}(\cos \theta)^{b^{*}} Y_{m}^{m}(\Omega),
$$

where we have set $a=a^{*}=m$ and $b=b^{*}$. Using the previously determined values of $b^{*}$, we obtain the results displayed in Table II. Notice that the generic behavior, for all values of $l \geq m+2$, is $r Q \propto u^{-l}$. It can be checked that repeated action of $\cos \theta$ on $Y_{m}^{m}(\Omega)$ generates spherical-harmonic functions of higher degree (see Ref. [50], Sec. 12.9). If $l-m$ is even, $(\cos \theta)^{l-m-2} Y_{m}^{m}$ can be expressed as a linear superposition of $Y_{m}^{m}, Y_{m+2}^{m}$, $Y_{m+4}^{m}, \ldots$, and $Y_{l-2}^{m}$. If $l-m$ is odd, on the other hand, $(\cos \theta)^{l-m-2} Y_{m}^{m}$ can be expressed as a linear superposition of $Y_{m+1}^{m}, Y_{m+3}^{m}, \ldots$, and $Y_{l-2}^{m}$. The generic situation at future null infinity therefore corresponds to a superposition of modes that all come with the same falloff behavior. The results derived here are identical to those reviewed at the beginning of the section and displayed in 
TABLE II: Behavior of $r Q\left(t, \boldsymbol{x} ; r^{\prime}\right)$ at future null infinity.

\begin{tabular}{ll}
\hline \hline If $\ldots$ & $r Q(v=\infty)$ is proportional to $\ldots$ \\
\hline$l=m$ & $\left(r^{\prime}\right)^{m} Y_{m}^{m}(\Omega) / u^{m+2}$ \\
$l=m+1$ & $\left(r^{\prime}\right)^{m+1} \cos \theta Y_{m}^{m}(\Omega) / u^{m+3}$ \\
$l \geq m+2$ & $\left(r^{\prime}\right)^{l-2}(\cos \theta)^{l-m-2} Y_{m}^{m}(\Omega) / u^{l}$ \\
\hline \hline
\end{tabular}

Eq. (1.8).

The description of the radiative falloff of a scalar field presented in this section is much more complicated than what was presented in Sec. V. Since the method of calculation is identical in both cases, the complexity encountered here clearly originates from our use of spheroidal coordinates, which obscure the underlying spherical symmetry of the problem. Since the spheroidal coordinates are directly related to the Boyer-Lindquist coordinates used to describe the Kerr metric, the analysis presented here suggests strongly that the apparent complexity of radiative falloff in Kerr must be attributed to the coordinate system, and not to the fact that the spacetime possesses angular momentum and higher multipole moments.

\section{DIRECT COMPARISON BETWEEN SPHEROIDAL AND SPHERICAL DESCRIPTIONS OF INITIAL DATA}

In this section we attempt a direct comparison between the different pictures of radiative falloff that emerge after using spherical coordinates $(r, \theta, \phi)$ on the one hand, and spheroidal coordinates $(\hat{r}, \hat{\theta}, \phi)$ on the other. Notice that as in Sec. I, we now use different notations for the two coordinate systems. We shall also use the short hands $\Omega=(\theta, \phi)$ and $\hat{\Omega}=(\hat{\theta}, \phi)$. For the purpose of this comparison we will restrict our attention to the field's falloff behavior near future timelike infinity.

The results derived in Sec. VI - see Table I show that when the field's initial data is of the form $\hat{C}(\hat{r}) Y_{l}^{m}(\hat{\Omega})$ when expressed in spheroidal coordinates, the evolution produces a dominant late-time mode that is either $Y_{m}^{m}(\hat{\Omega})$ if $l-m$ is even, or $Y_{m+1}^{m}(\hat{\Omega})$ if $l-m$ is odd; mode conversion preserves the parity of the original mode. In the generic situation (for $l \geq m+2$ ), this latetime mode comes with a falloff behavior that is either $t^{-(l+m+1)}$ if $l-m$ is even, or $t^{-(l+m+2)}$ if $l-m$ is odd. These results contradict a naive expectation according to which a $Y_{m}^{m}$ mode should decay as $t^{-(2 m+3)}$, while a $Y_{m+1}^{m}$ mode should decay as $t^{-(2 m+5)}$. This expectation comes from a description in terms of spherical coordinates, according to which a $Y_{l}^{m}$ mode has a falloff behavior given by $t^{-(2 l+3)}$; it is verified for the special cases $l=m$ and $l=m+1$, but not for the generic situation.

To shed some light on this contradiction, let us first consider initial data of the form $\Psi(0, \boldsymbol{x})=\hat{C}(\hat{r}) Y_{l}^{m}(\hat{\Omega})$ with $l-m$ even. We imagine transforming the right-hand side of this expression into spherical coordinates $(r, \theta, \phi)$. This would bring the field's initial data into the general form

$$
\Psi(0, \boldsymbol{x})={ }_{m} C(r) Y_{m}^{m}(\Omega)+{ }_{m+2} C(r) Y_{m+2}^{m}(\Omega)+\cdots,
$$

in which the radial functions ${ }_{m} C(r),{ }_{m+2} C(r)$, and so on, are determined by the transformation. We then isolate the dominant mode of lowest degree and substitute it into Eq. (5.4) to calculate $S(t, \boldsymbol{x})$, which gives the field's late-time behavior. To compute this we first evaluate $Q\left(t, \boldsymbol{x} ; r^{\prime}\right)$ as in Eq. (5.12), which we rewrite as

$$
Q\left(t, \boldsymbol{x} ; r^{\prime}\right) \propto \frac{\left(r r^{\prime}\right)^{m}}{t^{2 m+3}}\left(1+\frac{r^{\prime 2}}{t^{2}}\right)^{m+2} Y_{m}^{m}(\Omega),
$$

where we ignore all corrections that come with higher powers of $r$. Substituting this into Eq. (5.6) yields

$$
S(t, \boldsymbol{x}) \propto r^{m} Y_{m}^{m}(\Omega) \sum_{k=0}^{m+2} \frac{(m+2) !}{k !(m+2-k) !} \frac{{ }_{m} C_{m+2 k}}{t^{2 m+3+2 k}},
$$

where

$$
{ }_{m} C_{m+2 k} \equiv \int{ }_{m} C(r) r^{m+2 k+2} d r
$$

are moments of the relevant radial function.

Under normal circumstances the sum of Eq. (7.3) would be dominated by the term $k=0$ and we would have $S \propto{ }_{m} C_{m} r^{m} Y_{m}^{m}(\Omega) / t^{2 m+3}$, which goes according to the naive expectation. If, however, the first moment ${ }_{m} C_{m}$ were to vanish, then $S$ would be found to decay faster, and the naive expectation would be invalid. As a matter of fact, if a number of moments were to vanish, so that

$$
{ }_{m} C_{m}={ }_{m} C_{m+2}=\cdots={ }_{m} C_{l-4}=0,
$$

then the sum of Eq. (7.4) would start at $k=\frac{1}{2}(l-m)-1$ and we would have

$$
S(t, \boldsymbol{x}) \propto{ }_{m} C_{l-2} r^{m} Y_{m}^{m}(\Omega) / t^{l+m+1},
$$

in agreement with the generic results displayed in Table I. We see that reconciliation between the results of Sec. V and VI relies on the cancellations of Eq. (7.5): the naive expectation is false because the correct number of moments happen to vanish.

Before we investigate whether such a scenario is just too far fetched, let us state the conditions that produce a reconciliation when $l-m$ is odd. Here Eq. (7.1) must be replaced by

$$
\Psi(0, \boldsymbol{x})={ }_{m+1} C(r) Y_{m+1}^{m}(\Omega)+{ }_{m+3} C(r) Y_{m+3}^{m}(\Omega)+\cdots,
$$

and Eq. (7.3) becomes

$$
\begin{aligned}
S(t, \boldsymbol{x}) \propto & r^{m+1} Y_{m+1}^{m}(\Omega) \sum_{k=0}^{m+3} \frac{(m+3) !}{k !(m+3-k) !} \\
& \times \frac{m+1}{t^{2 m+5+2 k}},
\end{aligned}
$$


where ${ }_{m+1} C_{m+1+2 k}$ are moments of the radial function ${ }_{m+1} C(r)$. Normally the sum would be dominated by the $k=0$ term and we would have $S \propto$ ${ }_{m+1} C_{m+1} r^{m+1} Y_{m+1}^{m}(\Omega) / t^{2 m+5}$, in accordance with the naive expectation. But if

$$
{ }_{m+1} C_{m+1}={ }_{m+1} C_{m+3}=\cdots={ }_{m+1} C_{l-4}=0,
$$

then the sum of Eq. (7.4) would start at $k=\frac{1}{2}(l-m-$ 1) -1 and we would have

$$
S(t, \boldsymbol{x}) \propto{ }_{m+1} C_{l-2} r^{m+1} Y_{m+1}^{m}(\Omega) / t^{l+m+2},
$$

in agreement with the generic results displayed in Table I.

How do Eqs. (7.5) and (7.9) get enforced, given that the radial function $\hat{C}(\hat{r})$, in the original spheroidal coordinates, is arbitrary? While we cannot give a complete answer to this question, we can identify the basic mechanism by examining a specific example. Let us consider the initial data

$$
\Psi(0, \boldsymbol{x})=\hat{C}(\hat{r}) P_{4}(\cos \hat{\theta}),
$$

in which the angular function is a Legendre polynomial of order 4 . In this expression we substitute $\hat{r}(x, y, z)$ obtained from solving $\hat{r}^{4}-\left(x^{2}+y^{2}+z^{2}-e^{2}\right) \hat{r}^{2}-e^{2} z^{2}=0$, which can be inferred from Eq. (6.1). We also substitute $\cos \hat{\theta}=z / \hat{r}(x, y, z)$ and thus obtain $\Psi(0, \boldsymbol{x})$ explicitly in terms of Cartesian coordinates. Then we make the replacements $x=r \sin \theta \cos \phi, y=r \sin \theta \sin \phi$, and $z=r \cos \theta$, and finally express $\Psi(0, \boldsymbol{x})$ in terms of spherical coordinates. The result is complicated, and we simplify it by expanding it in powers of $e^{2}$ and discarding terms of order $e^{8}$ and higher. When we decompose the resulting expression in terms of Legendre polynomials, we obtain a series of the form

$$
\Psi(0, \boldsymbol{x})={ }_{0} C(r) P_{0}(\cos \theta)+{ }_{2} C(r) P_{2}(\cos \theta)+\cdots,
$$

which is a special case of Eq. (7.1). The relevant part of this expression is the spherically-symmetric mode, and we find that

$$
\begin{aligned}
{ }_{0} C(r)= & \frac{e^{4}}{315}\left(\frac{\hat{C}^{\prime \prime}}{r^{2}}+5 \frac{\hat{C}^{\prime}}{r^{3}}-5 \frac{\hat{C}}{r^{4}}\right)-\frac{e^{6}}{1155}\left(\frac{\hat{C}^{\prime \prime \prime}}{r^{3}}\right. \\
& \left.+\frac{7}{3} \frac{\hat{C}^{\prime \prime}}{r^{4}}-10 \frac{\hat{C}^{\prime}}{r^{5}}+10 \frac{\hat{C}}{r^{6}}\right)+O\left(e^{8}\right),
\end{aligned}
$$

where a prime indicates differentiation with respect to $\hat{r}$ with the result evaluated at $\hat{r}=r$. We then see that

$$
{ }_{0} C_{0} \equiv \int{ }_{0} C(r) r^{2} d r=O\left(e^{8}\right),
$$

which follows from Eq. (7.13) by straightforward integration by parts - the boundary terms are zero because the arbitrary function $\hat{C}(\hat{r})$ has compact support. The fact that Eq. (7.13) produces a vanishing moment at order $e^{4}$ and $e^{6}$ is truly remarkable, and we can perhaps believe that this result will extend to higher orders. If so, we would conclude that ${ }_{0} C_{0}$ vanishes, and we would recover the appropriate special case of Eq. (7.5).

The lesson learned here is that while $\hat{C}(\hat{r})$ is indeed arbitrary, the transformation to spherical coordinates is sufficiently special that it always produces functions ${ }_{m} C(r)$ and ${ }_{m+1} C(r)$ whose first several moments vanish according to Eqs. (7.5) and (7.9). While we cannot describe how this happens in complete generality, the previous example gives us a vivid illustration of the remarkable mechanism at work.

\section{Acknowledgments}

This work was supported by the Natural Sciences and Engineering Research Council of Canada. Conversations with John Friedman and Alan Wiseman were greatly appreciated, and led to the material presented in Sec. VII.

\section{APPENDIX A: TWO-POINT FUNCTIONS}

Our task in this appendix is to derive the expression for $U\left(x, x^{\prime}\right)$ presented in Eq. (4.13), and to justify the inequality of Eq. (4.4). We recall some notation: The spacetime point $x$ is decomposed as $(t, \boldsymbol{x})$, and similarly, $x^{\prime}=\left(t^{\prime}, \boldsymbol{x}^{\prime}\right)$ and $x^{\prime \prime}=\left(t^{\prime \prime}, \boldsymbol{x}^{\prime \prime}\right)$; we set $r=|\boldsymbol{x}|, r^{\prime}=\left|\boldsymbol{x}^{\prime}\right|$, $R=\left|\boldsymbol{x}-\boldsymbol{x}^{\prime}\right|$, and $\Delta t=t-t^{\prime}$. The methods employed here originate from Ref. [43], but this particular implementation comes from Ref. [47]; additional details are presented in Ref. [44].

To compute $U\left(x, x^{\prime}\right)$ we substitute Eq. (3.8) into Eq. (4.5) and integrate over $d t^{\prime \prime}$ to obtain

$$
U\left(x, x^{\prime}\right)=\frac{1}{2 \pi} \int \frac{\delta\left(\Delta t-\left|\boldsymbol{x}-\boldsymbol{x}^{\prime \prime}\right|-\left|\boldsymbol{x}^{\prime}-\boldsymbol{x}^{\prime \prime}\right|\right)}{\left|\boldsymbol{x}^{\prime \prime}\right|\left|\boldsymbol{x}-\boldsymbol{x}^{\prime \prime}\right|\left|\boldsymbol{x}^{\prime}-\boldsymbol{x}^{\prime \prime}\right|} d^{3} x^{\prime \prime} .
$$

To eliminate the $\delta$-function we align the $z$ axis with the direction of $\boldsymbol{x}-\boldsymbol{x}^{\prime}$ and set $\boldsymbol{x}=(x, y, z), \boldsymbol{x}^{\prime}=$ $(x, y, z-R)$. We then perform the coordinate transformation $\boldsymbol{x}^{\prime \prime}(s, \alpha, \beta)$ given by

$$
\begin{aligned}
x^{\prime \prime} & =x+\frac{1}{2} \sqrt{s^{2}-R^{2}} \sin \alpha \cos \beta, \\
y^{\prime \prime} & =y+\frac{1}{2} \sqrt{s^{2}-R^{2}} \sin \alpha \sin \beta, \\
z^{\prime \prime} & =z-\frac{1}{2} R+\frac{1}{2} s \cos \alpha .
\end{aligned}
$$

In terms of the new coordinates $(s, \alpha, \beta)$ we have $\mid \boldsymbol{x}-$ $\boldsymbol{x}^{\prime \prime}\left|=\frac{1}{2}(s-R \cos \alpha),\right| \boldsymbol{x}^{\prime}-\boldsymbol{x}^{\prime \prime} \mid=\frac{1}{2}(s+R \cos \alpha)$, and $d^{3} x^{\prime \prime}=\frac{1}{8}(s-R \cos \alpha)(s+R \cos \alpha) \sin \alpha d \alpha d \beta d s$. After substituting these results into Eq. (A1) and integrating over $d s$, we obtain

$$
U\left(x, x^{\prime}\right)=\frac{1}{4 \pi} \int \frac{1}{\left|\boldsymbol{x}^{\prime \prime}\right|} \sin \alpha d \alpha d \beta,
$$


where $\boldsymbol{x}^{\prime \prime}$ now stands for the vector $\boldsymbol{x}^{\prime \prime}(\Delta t, \alpha, \beta)$.

To evaluate the integral of Eq. (A3) we set $\boldsymbol{x}^{\prime \prime}=\boldsymbol{\eta}-\overline{\boldsymbol{\eta}}$ and express the vectors $\boldsymbol{\eta}$ and $\overline{\boldsymbol{\eta}}$ in terms of their ellipsoidal coordinates $(\Delta t, \alpha, \beta)$ and $(\bar{s}, \bar{\alpha}, \bar{\beta})$, respectively. We have $\eta^{x}=$ $\frac{1}{2} \sqrt{\Delta t^{2}-R^{2}} \sin \alpha \cos \beta, \eta^{y}=\frac{1}{2} \sqrt{\Delta t^{2}-R^{2}} \sin \alpha \sin \beta$, $\eta^{z}=\frac{1}{2} \Delta t \cos \alpha, \bar{\eta}^{x}=\frac{1}{2} \sqrt{\bar{s}^{2}-R^{2}} \sin \bar{\alpha} \cos \bar{\beta} \equiv-x$, $\bar{\eta}^{y}=\frac{1}{2} \sqrt{\bar{s}^{2}-R^{2}} \sin \bar{\alpha} \sin \bar{\beta} \equiv-y$, and $\bar{\eta}^{z}=\frac{1}{2} \bar{s} \cos \bar{\alpha} \equiv$ $-z+\frac{1}{2} R$. From the last set of equations we infer $\bar{s}=r+r^{\prime}$. We then invoke the addition theorem in ellipsoidal coordinates [51] and express $1 /\left|\boldsymbol{x}^{\prime \prime}\right|$ as

$$
\begin{aligned}
\frac{1}{|\boldsymbol{\eta}-\overline{\boldsymbol{\eta}}|}= & \frac{8 \pi}{R} \sum_{l=0}^{\infty} \sum_{m=-l}^{l}(-1)^{m} \frac{(l-m) !}{(l+m) !} P_{l}^{m}\left(s_{<} / R\right) \\
& \times Q_{l}^{m}\left(s_{>} / R\right) Y_{l}^{m *}(\bar{\alpha}, \bar{\beta}) Y_{l}^{m}(\alpha, \beta), \quad
\end{aligned}
$$

where $s_{<}=\min \left(\Delta t, r+r^{\prime}\right), s_{>}=\max \left(\Delta t, r+r^{\prime}\right)$, and $P_{l}^{m}$ and $Q_{l}^{m}$ are associated Legendre functions. Substituting this into Eq. (A3) we find that integration over the spherical harmonics $Y_{l}^{m}(\alpha, \beta)$ gives zero unless $l$ and $m$ are both zero. The only relevant Legendre functions are therefore $P_{0}(a)=1$ and $Q_{0}(a)=\frac{1}{2} \ln [(a+1) /(a-1)]$. Gathering the results, we have that

$$
U\left(x, x^{\prime}\right)=\frac{1}{R} \ln \frac{r+r^{\prime}+R}{r+r^{\prime}-R}
$$

if $\Delta t<r+r^{\prime}$, and

$$
U\left(x, x^{\prime}\right)=\frac{1}{R} \ln \frac{\Delta t+R}{\Delta t-R}
$$

if $\Delta t>r+r^{\prime}$. This last result was previously quoted in Eq. (4.13).

By substituting Eqs. (A5) and (A6) into Eq. (4.11) we find that

$$
U_{a}\left(x, x^{\prime}\right)=-2 \frac{x^{a} / r+x^{\prime a} / r^{\prime}}{\left(r+r^{\prime}\right)^{2}-R^{2}} \theta\left(r+r^{\prime}-\Delta t\right),
$$

which implies that $U_{a}\left(x, x^{\prime}\right)=0$ for $\Delta t>r+r^{\prime}$. This statement carries over to all other members of the sequence, and we conclude that $U\left(x, x^{\prime}\right)$ is the only member that does not vanish in this interval.

The two-point function $U\left(x, x^{\prime}\right)$ is the first member of a sequence $U_{N}\left(x, x^{\prime}\right)$ that arises as a result of expressing the gravitational potentials $\Phi\left(\boldsymbol{x}^{\prime \prime}\right)$ and $\boldsymbol{A}\left(\boldsymbol{x}^{\prime \prime}\right)$ as multipole expansions in powers of $1 /\left|\boldsymbol{x}^{\prime \prime}\right|$. Such expansions are meaningful only if

$$
\left|\boldsymbol{x}^{\prime \prime}\right|>\mathcal{R},
$$

where $\mathcal{R}$ denotes the maximum radius of the matter distribution. The two-point functions, therefore, are defined only if the condition of Eq. (A8) can be met. Since $\boldsymbol{x}^{\prime \prime}$, as defined by Eq. (A2) with $s$ set equal to $\Delta t$, depends on the relative positions of $x$ and $x^{\prime}$, we must examine under what circumstances Eq. (A8) can be expected to hold. As we shall see, Eq. (A8) can be replaced by a sequence of stronger inequalities that lead to Eq. (4.4). In other words, Eq. (A8) will be enforced, and the two-point functions $U_{N}\left(x, x^{\prime}\right)$ will be meaningful, so long as

$$
\Delta t>r+3 r^{\prime}+2 \mathcal{R},
$$

which is just a restatement of Eq. (4.4). For concreteness we shall assume that $r>r^{\prime}$. Notice that if Eq. (A9) holds, then $\Delta t$ is larger than $r+r^{\prime}, U\left(x, x^{\prime}\right)$ is given by Eq. (A6), and all other members of the sequence vanish.

We set $\boldsymbol{x}^{\prime \prime}=\boldsymbol{x}^{\prime}+\boldsymbol{\xi}$ and let the new vector $\boldsymbol{\xi}$ have components $\xi^{x}=\frac{1}{2} \sqrt{\Delta t^{2}-R^{2}} \sin \alpha \cos \beta$, $\xi^{y}=$ $\frac{1}{2} \sqrt{\Delta t^{2}-R^{2}} \sin \alpha \sin \beta$, and $\xi^{z}=\frac{1}{2}(R+\Delta t \cos \alpha)$. This vector has a length $\xi \equiv|\boldsymbol{\xi}|=\frac{1}{2}(\Delta t+R \cos \alpha)$ that satisfies

$$
\frac{1}{2}(\Delta t-R) \leq \xi \leq \frac{1}{2}(\Delta t+R) .
$$

On the other hand,

$$
\left|\xi-r^{\prime}\right| \leq\left|\boldsymbol{x}^{\prime \prime}\right| \leq \xi+r^{\prime},
$$

and we also have

$$
r-r^{\prime} \leq R \leq r+r^{\prime} .
$$

These inequalities will now be used to show that Eq. (A8) follows from Eq. (A9).

Equation (A9) can be re-expressed as $\Delta t-2\left(r^{\prime}+\mathcal{R}\right)>$ $r+r^{\prime}$. But $r+r^{\prime} \geq R$ by virtue of Eq. (A12), so we have $\Delta t-2\left(r^{\prime}+\mathcal{R}\right)>R$, which is equivalent to $r^{\prime}+\mathcal{R}<\frac{1}{2}(\Delta t-$ $R)$. But it follows from Eq. (A10) that $\frac{1}{2}(\Delta t-R) \leq \xi$, and we now have $r^{\prime}+\mathcal{R}<\xi$, or $\mathcal{R}<\xi-r^{\prime}$. This last result implies that $\xi-r^{\prime}>0$, and Eq. (A11) states that $\xi-r^{\prime} \leq\left|\boldsymbol{x}^{\prime \prime}\right|$. So finally we recover $\mathcal{R}<\left|\boldsymbol{x}^{\prime \prime}\right|$, and we conclude that Eq. (A8) can indeed be deduced from the stronger condition displayed in Eq. (A9).

\section{APPENDIX B: ANGULAR INTEGRATIONS}

In this Appendix we evaluate the integrals

$$
I_{p}(\Omega)=\frac{1}{4 \pi} \int(\cos \gamma)^{p} Y_{l}^{m}\left(\Omega^{\prime}\right) d \Omega^{\prime}
$$

introduced in Eq. (5.10), where $\cos \gamma=\cos \theta \cos \theta^{\prime}+$ $\sin \theta \sin \theta^{\prime} \cos \left(\phi-\phi^{\prime}\right)$. We start by recalling the wellknown property (see Ref. [50], Exercise 12.4.6) that $(\cos \gamma)^{p}$ can be expanded in terms of Legendre polynomials $P_{L}(\cos \gamma)$, with the sum over $L$ including even values only if $p$ is even, and odd values only if $p$ is odd; in either case the sum is limited by $L \leq p$. We combine this with the addition theorem for spherical harmonics (see Ref. [50], Sec. 12.8), which states that

$$
P_{L}(\cos \gamma)=\frac{4 \pi}{2 L+1} \sum_{M=-L}^{L} Y_{L}^{M *}\left(\Omega^{\prime}\right) Y_{L}^{M}(\Omega) .
$$


This identity implies that $P_{L}(\cos \gamma) Y_{l}^{m}\left(\Omega^{\prime}\right)$ integrated over $d \Omega^{\prime}$ is zero unless $L=l$. It follows that the only relevant part of the decomposition of $(\cos \gamma)^{p}$ in Legendre polynomials is

$$
(\cos \gamma)^{p}=\cdots+\frac{2^{l}(2 l+1) p !\left[\frac{1}{2}(p+l)\right] !}{(p+l+1) !\left[\frac{1}{2}(p-l)\right] !} P_{l}(\cos \gamma)+\cdots,
$$

and this will actually be present in the decomposition if $p \geq l$ and has the same parity as $l$. The values of $p$ for which $I_{p}(\Omega)$ is nonzero are therefore limited to the set $\{l, l+2, l+4, \cdots\}$. For these, substitution of Eq. (B2) into Eq. (B3), and substitution of that into Eq. (B1), allows the integration over $d \Omega^{\prime}$ to be carried out. This gives

$$
I_{p}(\Omega)=\frac{2^{l} p !\left[\frac{1}{2}(p+l)\right] !}{(p+l+1) !\left[\frac{1}{2}(p-l)\right] !} Y_{l}^{m}(\Omega) .
$$

This agrees with Eq. (5.11), where we have set $p=l+2 n$.

\section{APPENDIX C: MORE ANGULAR INTEGRATIONS}

In this appendix we give sketchy evaluations of the integrals

$$
I_{a b}\left(r^{\prime}, \Omega\right)=\frac{1}{4 \pi} \int\left(r^{\prime 2}+e^{2} C^{2}\right) S^{a} C^{b} Y_{l}^{m}\left(\Omega^{\prime}\right) d \Omega^{\prime}
$$

introduced in Eq. (6.8); we recall the definitions $S \equiv$ $\sin \theta \sin \theta^{\prime} \cos \left(\phi-\phi^{\prime}\right)$ and $C \equiv \cos \theta^{\prime}$. We are interested mostly in determining the smallest values of $a$ and $b$ such that $I_{a b}$ does not vanish; we denote these $a^{*}$ and $b^{*}$, respectively. We shall derive the general form of $I_{a^{*} b^{*}}\left(r^{\prime}, \Omega\right)$ but leave all numerical coefficients undetermined.

The integral $I_{a b}$ will be nonzero if either $S^{a} C^{b} Y_{l}^{m}$ or $S^{a} C^{b+2} Y_{l}^{m}$ contains a term proportional to $Y_{0}^{0}$ when decomposed into spherical harmonics. The action of $S$ is given schematically by (see Ref. [50], Sec. 12.9)

$$
\begin{aligned}
S Y_{l}^{m}= & \sin \theta e^{i \phi}\left[() Y_{l-1}^{m-1}+() Y_{l+1}^{m-1}\right] \\
& +\sin \theta e^{-i \phi}\left[() Y_{l-1}^{m+1}+() Y_{l+1}^{m+1}\right],
\end{aligned}
$$

where the empty brackets denote undetermined numerical coefficients; acting with $S$ therefore lowers and raises $m$ by one unit. On the other hand, the action of $C$ is given schematically by (see Ref. [50], Sec. 12.9)

$$
C Y_{l}^{m}=() Y_{l-1}^{m}+() Y_{l+1}^{m},
$$

and it leaves $m$ unchanged. It is clear that to reduce $m$ to zero we need a minimum number $m$ of repeated applications of the operator $S$. We therefore have $a^{*}=m$, and it easy to check that $S^{m} Y_{l}^{m}$ must have the schematic form

$$
\begin{aligned}
S^{m} Y_{l}^{m}\left(\Omega^{\prime}\right)= & Y_{m}^{m}(\Omega) \sum_{s=0}^{m}() Y_{l-m+2 s}^{0}\left(\Omega^{\prime}\right) \\
& + \text { terms in } Y^{2}, Y^{4}, \cdots, Y^{2 m}
\end{aligned}
$$

the presence of $Y_{m}^{m}(\Omega)$ comes from the fact that $m$ applications of $S$ generate the factor $\left(\sin \theta e^{i \phi}\right)^{m} \propto Y_{m}^{m}(\Omega)$. The terms that do not involve $Y^{0}$ disappear after integration over $d \Omega^{\prime}$, and we obtain

$$
I_{a^{*} b}=Y_{m}^{m}(\Omega) \sum_{s=0}^{m}() \int\left(r^{2}+a^{2} C^{2}\right) C^{b} Y_{l-m+2 s}^{0}\left(\Omega^{\prime}\right) d \Omega^{\prime} .
$$

Repeated application of $C$ now gives

$$
C^{b} Y_{l-m+2 s}^{0}\left(\Omega^{\prime}\right)=\sum_{s^{\prime}=0}^{b}() Y_{l-m-b+2 s+2 s^{\prime}}^{0}\left(\Omega^{\prime}\right),
$$

and substitution into Eq. (C5) gives

$$
\begin{aligned}
I_{a^{*} b}= & Y_{m}^{m}(\Omega) \sum_{s=0}^{m}\left\{() e^{2} \int Y_{l-m-b-2+2 s}^{0}\left(\Omega^{\prime}\right) d \Omega^{\prime}\right. \\
& \left.+\sum_{s^{\prime}=0}^{b}\left[() r^{\prime 2}+() e^{2}\right] \int Y_{l-m-b+2 s+2 s^{\prime}}^{0}\left(\Omega^{\prime}\right) d \Omega^{\prime}\right\},
\end{aligned}
$$

after some reorganization of the sum over $s^{\prime}$ and elimination of integrals that vanish automatically.

We are seeking the smallest value of $b$ such that $I_{a^{*} b}$, as now given by Eq. (C7), does not vanish. Let us consider a few special cases.

If $l=m$, then the first integral of Eq. (C7) is nonzero when $b=2 s-2$, and since $b$ cannot be negative, the smallest possible value of $b$ is 0 . For the second integral not to vanish we need $b=2 s+2 s^{\prime}$, and again the smallest possible value of $b$ is 0 . We conclude that $b^{*}=0$ when $l=m$, and that $I_{a^{*} b^{*}}$ has the schematic form $\left[() r^{\prime 2}+\right.$ $\left(\right.$ ) $\left.e^{2}\right] Y_{m}^{m}(\Omega)$.

If $l=m+1$, then the first integral of Eq. (C7) is nonzero when $b=2 s-1$, and the smallest allowed value of $b$ is now 1 . For the second integral we need $b=1+$ $2 s+2 s^{\prime}$, which gives rise to the same minimum value of 1 . We conclude that $b^{*}=1$ when $l=m+1$. In this case, $I_{a^{*} b^{*}}$ has the same schematic form as before, $\left[() r^{2}+() e^{2}\right] Y_{m}^{m}(\Omega)$.

If $l=m+2$, then the first integral of Eq. (C7) is nonzero when $b=2 s$, and the smallest allowed value of $b$ is again 0 . For the second integral we need $b=2+2 s+2 s^{\prime}$, which gives rise to a smallest allowed value of 2 . Since this value is larger than the first, we can ignore the second integral. We conclude that $b^{*}=0$ when $l=m+2$, and $I_{a^{*} b^{*}}=() e^{2} Y_{m}^{m}(\Omega)$.

If $l=m+3$, then the first integral of Eq. (C7) is nonzero when $b=2 s+1$, and the smallest allowed value of $b$ is now 1 . For the second integral we need $b=3+2 s+2 s^{\prime}$, which gives rise to a smallest allowed value of 3 . Since this value is larger than the first, we can once more ignore the second integral and conclude that $b^{*}=1$ when $l=$ $m+3$. This gives $I_{a^{*} b^{*}}=() e^{2} Y_{m}^{m}(\Omega)$.

This pattern continues for all other values of $l$. For $l \geq m-2$ we find that $b^{*}=l-m-2$, which gives rise to 
TABLE III: Smallest values of $a$ and $b$ such that the integral $I_{a b}\left(r^{\prime}, \Omega\right)$ is nonvanishing. The Table also displays the schematic form of $I_{a^{*} b^{*}}\left(r^{\prime}, \Omega\right)$, with empty brackets denoting undetermined numerical coefficients that depend on $l$ and $m$.

\begin{tabular}{llcc}
\hline \hline Case & $a^{*}$ & $b^{*}$ & $I_{a^{*} b^{*}}\left(r^{\prime}, \Omega\right)$ \\
\hline$l=m$ & $m$ & 0 & {$\left[() r^{\prime 2}+() e^{2}\right] Y_{m}^{m}(\Omega)$} \\
$l=m+1$ & $m$ & 1 & {$\left[() r^{\prime 2}+() e^{2}\right] Y_{m}^{m}(\Omega)$} \\
$l \geq m+2$ & $m$ & $l-m-2$ & ()$e^{2} Y_{m}^{m}(\Omega)$ \\
\hline \hline
\end{tabular}

$I_{a^{*} b^{*}}=() e^{2} Y_{m}^{m}(\Omega)$. Our results are summarized in Table III, and they were quoted in the paragraph following Eq. (6.8).
[1] R.H. Price, Phys. Rev. D 5, 2419 (1972); 2439 (1972).

[2] J.M. Bardeen and W.H. Press, J. Math. Phys. 14, 7 (1973).

[3] C. Gundlach, R.H. Price, and J. Pullin, Phys. Rev. D 49, 883 (1994),

[4] L. Barack, Phys. Rev. D 59, 044016 (1999); 044017 (1999).

[5] E.W. Leaver, Phys. Rev. D 34, 384 (1986).

[6] E.S.C. Ching, P.T. Leung, W.M. Suen, and K. Young, Phys. Rev. Lett. 74, 2414 (1995); Phys. Rev. D 52, 2118 (1995).

[7] N. Andersson, Phys. Rev. D 55, 468 (1997).

[8] S. Hod, Class. Quant. Grav. 18, 1311 (2001).

[9] N. Andersson, Y. Kojima, K.D. Kokkotas, Astrophys. J. 462, 855 (1996).

[10] N. Andersson and K.D. Kokkotas, Phys. Rev. Lett. 77, 4134 (1996); Mon. Not. Roy. Astron. Soc. 299, 1059 (1998).

[11] G. Allen, N. Andersson, K.D. Kokkotas, and B.F. Schutz, Phys. Rev. D 58, 124012 (1998).

[12] K. Tominaga, M. Saijo, and K.I. Maeda, Phys. Rev. D 60, 024004 (1999).

[13] Z. Andrade and R.H. Price, Phys. Rev. D 60, 104037 (1999).

[14] V. Pavlidou, K. Tassis, T.W. Baumgarte, and S.L. Shapiro, Phys. Rev. D 62, 084020 (2000).

[15] K. Glampedakis and N. Andersson, Class. Quant. Grav. 18, 1939 (2001).

[16] K.D. Kokkotas, T.A. Apostolatos, and N. Andersson, Mon. Not. Roy. Astron. Soc. 320 307, (2001).

[17] H. Sotani, K. Tominaga, and K.I. Maeda, Phys. Rev. D 65, 024010 (2002).

[18] C. Gundlach, R.H. Price, and J. Pullin, Phys. Rev. D 49, 890 (1994).

[19] L.M. Burko and A. Ori, Phys. Rev. D 56, 7820 (1997).

[20] R.L. Marsa and M.W. Choptuik, Phys. Rev. D 54, 4929 (1996).

[21] P.R. Brady, C.M. Chambers, W. Krivan, and P. Laguna, Phys. Rev. D 55, 7538 (1997).

[22] P.R. Brady, C.M. Chambers, W.G. Laarakkers, and E. Poisson, Phys. Rev. D 60, 064003 (1999).

[23] J.S.F. Chan and R.B. Mann, Phys. Rev. D 557546 (1997); 59064025 (1999).

[24] G.T. Horowitz and V.E. Hubeny, Phys. Rev. D 62, 024027 (2000).

[25] W. G. Laarakkers and E. Poisson, Phys. Rev. D 64, 084008 (2001).

[26] B. Wang, E. Abdalla, and R. B. Mann, hep-th/0107243.
[27] B. Wang, C. Molina, and E. Abdalla, Phys. Rev. D 63, 084001 (2001).

[28] S. Hod and T. Piran, Phys. Rev. D 58, 024017 (1998); 024018 (1998); 024019 (1998).

[29] S. Hod and T. Piran, Phys. Rev. D 58, 044018 (1998).

[30] R. G. Cai and A Wang, Gen. Rel. Grav. 31, 1367 (1999).

[31] H. Koyama and A. Tomimatsu, Phys. Rev. D 63, 064032 (2001); 64, 044014 (2001).

[32] R. Moderski and M. Rogatko, Phys. Rev.D 63, 084014 (2001); 64, 044024 (2001).

[33] S. Hod, Phys. Rev. D 58, 104022 (1998).

[34] L. Barack and A. Ori, Phys. Rev. Lett. 82, 4388 (1999).

[35] L. Barack and A. Ori, Phys. Rev. D 60, 124005 (1999).

[36] L. Barack, Phys. Rev. D 61, 024026 (2000).

[37] S. Hod, Phys. Rev. D 61, 024033 (2000); 064018 (2000).

[38] S. Hod, Phys. Rev. Lett. 84, 10 (2000).

[39] W. Krivan, P. Laguna, and P. Papadopoulos, Phys. Rev. D 54, 4728 (1996).

[40] W. Krivan, P. Laguna, P. Papadopoulos, and N. Andersson, Phys. Rev. D 56, 3395 (1997).

[41] W. Krivan, Phys. Rev. D 60, 101501 (1999).

[42] S. Hod, Phys. Rev. D 60, 104053 (1999).

[43] B.S. DeWitt and C.M. DeWitt, Physics (Long Island City, N.Y.) 1, 3 (1964).

[44] M.J. Pfenning and E. Poisson, Phys. Rev. D 65, 084001 (2002).

[45] R.M. Wald, General relativity (University of Chicago Press, Chicago, 1984), Sec. 4.4.

[46] F.A.E. Pirani, in Lectures on general relativity, edited by A. Trautman, F.A.E. Pirani, and H. Bondi (PrenticeHall, Englewood Cliffs, 1964).

[47] A.G. Wiseman (unpublished). Wiseman's unpublished calculations were presented at the Second Capra Meeting on Radiation Reaction, in Dublin, Ireland (1999). The proceedings of this conference, which contain Wiseman's presentation, can be found at http://www.lsc-group.phys. uwm.edu/ patrick/ ireland99/.

[48] B.S. DeWitt and R.W. Brehme, Ann. Phys. (N.Y.) 9, 220 (1960).

[49] J. Hadamard, Lectures on Cauchy's Problem in Linear Partial Differential Equations (Yale University Press, New Haven, 1923).

[50] G. Arfken, Mathematical Methods for Physicists (Academic Press, Orlando, 1985).

[51] T.M. MacRobert, Spherical Harmonics (Methuen, London, 1927), Chap. XI. 